\title{
LIGHT CURVES OF CORE-COLLAPSE SUPERNOVAE WITH SUBSTANTIAL MASS LOSS USING THE NEW OPEN-SOURCE SUPERNOVA EXPLOSION CODE (SNEC)
}

\author{
Viktoriya Morozova ${ }^{1}$, Anthony L. Piro ${ }^{2}$, Mathieu Renzo ${ }^{1,3}$, Christian D. Ott ${ }^{1}$, \\ Drew Clausen ${ }^{1}$, Sean M. Couch ${ }^{1}$, Justin Ellis ${ }^{1}$, and Luke F. Roberts ${ }^{1}$ \\ ${ }^{1}$ TAPIR, Walter Burke Institute for Theoretical Physics, MC 350-17, California Institute of Technology, Pasadena, CA 91125, USA; morozvs@tapir.caltech.edu \\ ${ }^{2}$ Carnegie Observatories, 813 Santa Barbara Street, Pasadena, CA 91101, USA \\ ${ }^{3}$ Dipartimento di Fisica "Enrico Fermi," University of Pisa, I-56127 Pisa, Italy \\ Received 2015 May 25; accepted 2015 October 15; published 2015 November 17
}

\begin{abstract}
We present the SuperNova Explosion Code (SNEC), an open-source Lagrangian code for the hydrodynamics and equilibrium-diffusion radiation transport in the expanding envelopes of supernovae. Given a model of a progenitor star, an explosion energy, and an amount and distribution of radioactive nickel, SNEC generates the bolometric light curve, as well as the light curves in different broad bands assuming blackbody emission. As a first application of SNEC, we consider the explosions of a grid of $15 M_{\odot}$ (at zero-age main sequence, ZAMS) stars whose hydrogen envelopes are stripped to different extents and at different points in their evolution. The resulting light curves exhibit plateaus with durations of $\sim 20-100$ days if $\gtrsim 1.5-2 M_{\odot}$ of hydrogen-rich material is left and no plateau if less hydrogen-rich material is left. If these shorter plateau lengths are not seen for SNe IIP in nature, it suggests that, at least for ZAMS masses $\lesssim 20 M_{\odot}$, hydrogen mass loss occurs as an all or nothing process. This perhaps points to the important role binary interactions play in generating the observed mass-stripped supernovae (i.e., Type $\mathrm{Ib} / \mathrm{c}$ events). These light curves are also unlike what is typically seen for SNe IIL, arguing that simply varying the amount of mass loss cannot explain these events. The most stripped models begin to show doublepeaked light curves similar to what is often seen for $\mathrm{SNe} \mathrm{IIb}$, confirming previous work that these supernovae can come from progenitors that have a small amount of hydrogen and a radius of $\sim 500 R_{\odot}$.
\end{abstract}

Key words: hydrodynamics - radiative transfer - supernovae: general

\section{INTRODUCTION}

During the last half century the number of observed supernovae $(\mathrm{SNe})$ has increased exponentially (Minkowski 1964; Cappellaro 2014). Much of this progress has been fueled by recent surveys, such as the Lick Observatory Supernova Search (LOSS, Leaman et al. 2011; Smith et al. 2011), the Sloan Digital Sky Survey (Frieman et al. 2008), and the Palomar Transient Factory (PTF, Rau et al. 2009). In addition to providing more complete and detailed samples of wellknown classes of SNe (Type Ia, Ib/c, II), these surveys have found a wide range of previously unknown explosive events, from superluminous SNe (Quimby et al. 2011) to rapid SN-like transients (Kasliwal et al. 2010, 2012; Perets et al. 2010; Foley et al. 2013; Inserra et al. 2015). This has opened our eyes to the broader range of astrophysical explosions that can exist in nature.

Progress in explosive, transient observations has been closely followed by progress in analytic and numerical light curve modeling. For example, for SNe IIP, this has ranged from analytic scalings (Arnett 1980; Chugai 1991; Popov 1993) to detailed numerical works (e.g., Litvinova \& Nadezhin 1983; Chieffi et al. 2003; Young 2004; Kasen \& Woosley 2009; Bersten et al. 2011; Dessart et al. 2013). These investigations focused on understanding the general imprint of progenitor characteristics (mass, radius, abundance and mixing of ${ }^{56} \mathrm{Ni}$, etc.) on the shape and luminosity of SN light curves. In other cases, detailed comparisons have been made between specific SNe II and numerical models (Arnett 1988; Shigeyama et al. 1988; Woosley 1988; Utrobin 1993 for SN 1987A, Nomoto et al. 1993; Bartunov et al. 1994; Shigeyama et al. 1994; Young et al. 1995; Blinnikov et al. 1998 for SN 1993J, Baklanov et al. 2005; Utrobin 2007 for SN 1999em).
The combination of growing samples of $\mathrm{SNe}$ and other previously unknown transients has motivated us to develop a new code for numerical studies of the light curves of SN and SN-like explosions. Called the SuperNova Explosion Code $(\mathrm{SNEC})$, this general purpose code will allow the user to take a stellar model (or other ad-hoc density profile with other thermodynamic and compositional information), input energy to generate an explosion, follow the hydrodynamic response, and produce light curves. The current iteration of SNEC is spherically symmetric (1D), and uses Lagrangian hydrodynamics and equilibrium-diffusion (one-temperature) radiation transport. It also follows other basic physics needed for light curves such as ionization and heating from ${ }^{56} \mathrm{Ni}$.

In terms of complexity and amount of physics included, SNEC is at a somewhat intermediate position compared with existing SN light curve codes. The current state-of-the-art are multi-group radiation-hydrodynamic codes (as in Blinnikov \& Bartunov 1993; Moriya et al. 2011) and line-transfer radiative transfer codes that assume homologous expansion and either make the local thermodynamic equlibrium approximation (LTE; e.g., Kasen \& Woosley 2009) or are fully non-LTE (e.g., Hillier \& Dessart 2012). SNEC bridges the gap between these codes and analytical investigations, e.g., those of Arnett (1980), Chugai (1991), and Popov (1993), and the more recent ones of Nakar \& Sari (2010), Goldfriend et al. (2014), and Nakar et al. (2015). Our work is very much in the same spirit as the works of Bersten et al. (2011) and Ergon et al. (2015). A crucial aspect of SNEC is that unlike these other codes, it is open source and publicly available. ${ }^{4}$ This will make light curve modeling accessible and reproducible for the broader

4 http://stellarcollapse.org/SNEC 
community. It can be used for a wide range of studies, from generating typical SN light curves as an educational tool to making light curves for novel explosion scenarios. Modeling explosions and light curves involves a wide range of physics and necessary approximations. Hence, making code available and results reproducible is crucial for the advancement of the field.

An important strength of SNEC is the ability to systematically and quickly explore changes in stellar properties to learn how they impact the resulting light curves. This is especially useful for investigating the underlying mechanisms behind the photometric diversity of SN II light curves, such as the SNe IIP (with nearly constant plateau luminosity for a period $\sim 100$ days past maximum, and the most common type), SNe IIL (with linearly declining magnitude), and $\mathrm{SNe}$ IIb (which show signatures of hydrogen present, but with a light curve generally more similar to $\mathrm{SNe} \mathrm{Ib}) .{ }^{5}$ In particular, the connection between SNe IIP and SNe IIL has long been a point of contention in the SN community. Early on, it was suggested by Barbon et al. (1979) and corroborated by Blinnikov \& Bartunov (1993) that the morphological differences might be explained by altering the envelope masses while keeping the explosion mechanism the same (however, see Swartz et al. 1991 for an alternative picture). In this explanation, SNe IIL would simply have less hydrogen-rich envelopes than SNe IIP. Nevertheless, SNe IIL must still have appreciable hydrogen present, otherwise they would become $\mathrm{SNe} \mathrm{Ib} / \mathrm{c}$ instead. This suggests that there may exist a continuous range of hydrogen mass stripping and thus a continuous range of events between canonical SNe IIP and IIL. Furthermore, SNe IIb have been inferred to have a small amount of hydrogen present $\quad\left(\sim 0.01-0.1 M_{\odot}\right.$, Woosley et al. 1994; Bersten et al. 2012; Nakar \& Piro 2014), and thus in principle with sufficient mass loss a transition should be seen all the way to $\mathrm{SNe} \mathrm{IIb}$. The question is whether additional ingredients are needed beyond just increased mass loss to reproduce these features.

Motivated by these questions, we investigate the mass-loss hypothesis for the origin of these SN II classes as a first application of SNEC. We use presupernova stellar models generated with the MESA code (Paxton et al. 2011, 2013). Besides providing excellent control in generating models and investigating mass stripping, MESA has been used for other light curve studies (e.g., Dessart et al. 2013), which allows us to compare directly as a further check of SNEC. Although we find that varying levels of hydrogen mass stripping shortens the plateau of the light curves, we conclude that simply varying the amount of mass loss alone cannot explain the full range of properties of SNe IIL. In the most mass stripped cases, we begin to see double-peaked light curves reminiscent of some $\mathrm{SNe}$ IIb, suggesting that this transition occurs more naturally. Further work will be needed for a more complete investigation of $\mathrm{SNe}$ IIb properties.

In Section 2, we describe SNEC in detail. We follow, in Section 3, with our study of massive stars with varying levels of stripping. In Section 4, we present the resulting SN light curves. We conclude in Section 5 with a summary of our findings and a discussion of future work. In Appendices A and B, we compare SNEC with two other codes.

\footnotetext{
5 We ignore SNe IIn for our study because they show clear signs of interaction, which is beyond the scope of this work.
}

\section{SNEC}

We describe SNEC with a focus on SN IIP light curve modeling. Although we anticipate that SNEC will continue to evolve and improve as it is utilized for new projects and more physics is added, the discussion below will provide some necessary background and summarize SNEC's general features. A more detailed description that also includes the finitedifference form of the equations and implementation details is available on the SNEC webpage, http://stellarcollapse. org/SNEC.

It is also important to compare SNEC with other SN light curve codes. Although below we focus on what is implemented in SNEC, in Appendices A and B we consider the work of Bersten et al. (2011), whose code has a similar level of complexity as SNEC, and Dessart et al. (2013), whose code performs a more detailed treatment of the radiative transfer. We find that both comparisons give satisfactory results with the main difference being the transition from the plateau to the ${ }^{56} \mathrm{Ni}$ tail found by Dessart et al. (2013). This disagreement likely reflects an intrinsic difference between equilibrium-diffusion radiation-hydrodynamics codes, such as SNEC, and linetransfer radiative-transfer codes such as CMFGEN used in Dessart et al. (2013).

\subsection{D Lagrangian LTE Radiation Hydrodynamics with Ionization}

Lagrangian hydrodynamics in spherical symmetry, supplemented with a radiation diffusion term, written to the first order in $v / c$ (see, e.g., Mihalas \& Mihalas 1984; Mezzacappa \& Bruenn 1993; Bersten 2010), results in a mass conservation equation (continuity equation),

$$
\frac{\partial r}{\partial m}=\frac{1}{4 \pi r^{2} \rho}
$$

an energy equation,

$$
\frac{\partial \epsilon}{\partial t}=\frac{P}{\rho} \frac{\partial \ln \rho}{\partial t}-4 \pi r^{2} Q \frac{\partial v}{\partial m}-\frac{\partial L}{\partial m}+\epsilon_{\mathrm{Ni}},
$$

and a momentum equation,

$$
\frac{\partial v}{\partial t}=-\frac{G m}{r^{2}}-4 \pi r^{2} \frac{\partial P}{\partial m}-4 \pi \frac{\partial\left(r^{2} Q\right)}{\partial m} .
$$

Here $m=\int_{0}^{r} 4 \pi r^{\prime 2} \rho\left(r^{\prime}\right) d r^{\prime}$ is the mass coordinate, $r$ is the radius, $t$ is the time, $\rho$ is the density, $\epsilon$ is the specific internal energy, $P$ is the pressure, $v=\partial r / \partial t$ is the velocity of the matter, $Q$ is the artificial viscosity, and $G$ is the gravitational constant. In Equation (2), $\epsilon_{\mathrm{Ni}}$ is the specific energy deposition rate due to the radioactive decay of ${ }^{56} \mathrm{Ni}$, which is equal to the time-dependent rate of energy release per gram of radioactive nickel $\epsilon_{\text {rad }}$, multiplied by the deposition function $d$, both defined below in Section 2.3 by Equations (14) and (15), respectively. The radiative luminosity $L$ is

$$
L=-\left(4 \pi r^{2}\right)^{2} \frac{\lambda a c}{3 \kappa} \frac{\partial T^{4}}{\partial m},
$$

where $a$ is the radiation constant, $c$ is the speed of light, $\lambda$ is the flux-limiter and $\kappa$ is the Rosseland mean opacity. For capturing shocks, we use a simple von Neumann-Richtmyer artificial 
viscosity (Von Neumann \& Richtmyer 1950)

$$
Q \equiv \begin{cases}C \rho(\partial v / \partial l)^{2} & \text { if } \partial v / \partial l<0 \\ 0 & \text { otherwise }\end{cases}
$$

where $l$ is an integer grid coordinate, and $C=2$. Following Bersten et al. (2011), we use the flux limiter of Levermore \& Pomraning (1981),

$$
\lambda=\frac{6+3 R}{6+3 R+R^{2}}
$$

where

$$
R=\frac{4 \pi r^{2}}{\kappa T^{4}}\left|\frac{\partial T^{4}}{\partial m}\right|
$$

We discretize in mass and time following the scheme of Mezzacappa \& Bruenn (1993). The mass conservation and momentum conservation equations are updated time-explicitly. For Equation (2), we use a semi-implicit scheme with an adjustable parameter $\theta$ in the discretization of the derivative $\partial L / \partial m$ that can vary from fully explicit $(\theta=0$, only the luminosity from the previous time step is used in the scheme) to fully implicit $(\theta=1$, only the luminosity at the next time step is used in the scheme). The derivative $\partial T^{4} / \partial m$ is linearized in $\delta T$. We use $\theta=1 / 2$ for all simulations presented in this paper. Using an initial guess for the temperature at the next time step, we iteratively solve for $\delta T$, inverting a tridiagonal matrix each time, until the fractional change in temperature is less than a set tolerance $\left(10^{-7}\right.$ in the current version of the SNEC). We do not take the dependence of the opacity $\kappa$ on temperature into account in the implicit update and rather use the opacity from the previous time step when solving for the temperature at the next step.

SNEC assumes local thermodynamical equilibrium (LTE), imposing the same temperature for radiation and matter. This assumption is not valid at shock breakout and during and after the transition phase from optically thick to optically thin ejecta. In SNe IIP, it is reliable only during the plateau phase of the light curve (see the discussions in Blinnikov \& Bartunov 1993; Bersten et al. 2011). However, the comparison performed by Bersten (2010) between her LTE code and a multi-group code suggests satisfactory agreement along the entire light curve.

As a boundary condition for Equation (3), we adopt $P=0$ at the center of the boundary cell, so half a grid cell outside the star. For Equation (2), we assume that the luminosity at the surface is equal to the luminosity at the closest interior grid point, i.e., that the diffusive term, $\partial L / \partial m$, at the outer boundary is equal to zero. At the inner boundary, we take the velocity and the bolometric luminosity to be zero. In the modeling of core-collapse SN light curves, the inner boundary is typically not at $m=0$ due to the presence of a neutron star (or a black hole), which is excluded from the grid. Setting the inner velocity to zero excludes any possibility for fallback of material onto the remnant in our models.

To close the system of hydrodynamic equations, we employ the analytic equation of state (EOS) given by Paczyński (1983), hereafter the Paczyński EOS. The Paczyński EOS contains contributions from radiation, ions, and electrons, and takes into account electron degeneracy approximately. We repeat some of our model calculations with the Helmholtz EOS (Timmes \& Arnett 1999; Timmes \& Swesty 2000), which includes a (tabulated) complete electron EOS, to test the approximations made in the Paczyński EOS. We find that differences between the Paczyński and Helmholtz EOS have negligible influence on the resulting light curves.

In order to account for recombination, we supplement the Paczyński EOS with a routine that solves the Saha equations in the non-degenerate approximation as proposed in Zaghloul et al. (2000). The set of Saha equations, together with the condition of charge neutrality and number conservation of nuclei of a given chemical element (enumerated by index $k$ ), may be combined into a single transcendental equation for the average charge $\overline{\mathbb{Z}}_{k}$ of element $k$ with atomic number $Z_{k}$ as

$$
1-\overline{\mathbb{Z}}_{k}\left(\sum_{i=1}^{Z_{k}} \frac{i \prod_{j=1}^{i} f_{k, j}}{\left(\overline{\mathbb{Z}}_{k} n_{k}\right)^{i}}\right)^{-1}\left[1+\sum_{i=1}^{Z_{k}} \frac{\prod_{j=1}^{i} f_{k, j}}{\left(\overline{\mathbb{Z}}_{k} n_{k}\right)^{i}}\right]=0
$$

with

$$
\begin{aligned}
f_{k, i+1} & =2 \frac{g_{k, i+1}}{g_{k, i}}\left[\frac{2 \pi m_{e} k_{\mathrm{B}} T}{h^{2}}\right]^{3 / 2} \exp \left(-\frac{I_{k, i}}{k_{\mathrm{B}} T}\right), \\
i & =0,1, \ldots,\left(Z_{k}-1\right),
\end{aligned}
$$

where $i$ is the number of the ionization state ( 0 corresponds to the neutral atom), $Z_{k}$ is the atomic number of element $k, n_{k}$ is the number density of element $k, g_{k, i}$ is the statistical weight of the $i$ th ionization state of element $k, I_{k, i}$ is the (positive) ionization energy for the ionization process $i \rightarrow(i+1), m_{e}$ is the electron rest mass, $h$ is Planck's constant and $k_{\mathrm{B}}$ is Boltzmann's constant. Equation (8) is solved iteratively at each call to the EOS, after which the ionization fractions $\alpha_{k, i}$ are found as

$$
\begin{aligned}
\alpha_{k, 0} & =\overline{\mathbb{Z}}_{k}\left(\sum_{i=1}^{\mathbb{Z}_{k}} \frac{i \prod_{j=1}^{i} f_{k, j}}{\left(\overline{\mathbb{Z}}_{k} n_{k}\right)^{i}}\right)^{-1}, \\
\alpha_{k, i+1} & =\frac{\alpha_{k, i}}{\overline{\mathbb{Z}}_{k} n_{k}} f_{k, i+1} \cdot
\end{aligned}
$$

Although one can consider as many elements as necessary at the expense of computational time, for the present work we focus on the ionization of hydrogen and helium. The specific internal energy is calculated as

$$
\epsilon=\epsilon_{\mathrm{ion}}+\epsilon_{\mathrm{el}}+\epsilon_{\mathrm{rad}}+\Delta \epsilon_{\mathrm{ion}},
$$

where $\epsilon_{\mathrm{ion}}, \epsilon_{\mathrm{el}}$, and $\epsilon_{\mathrm{rad}}$ are the contributions from ions, electrons, and radiation, respectively, and

$$
\Delta \epsilon_{\text {ion }}=\frac{1}{\rho} \sum_{k}\left[n_{k} \sum_{i=1}^{\mathbb{Z}_{k}}\left(\alpha_{k, i} \sum_{j=0}^{i-1} \boldsymbol{I}_{k, j}\right)\right]
$$

is the ionization energy whose zero point for each element $k$ is the neutral atom.

\subsection{Opacities}

The Rosseland mean opacity $\kappa$ is an essential input to our light curve models. In the high temperature regime $\left(10^{3.75} \mathrm{~K}<T<10^{8.7} \mathrm{~K}\right)$, we use the OPAL Type II opacity 
tables (Iglesias \& Rogers 1996) for solar metallicity $\left(Z_{\odot}=0.02\right.$ here). These tables allow for an increase in the mass fractions of two chosen metals (in our case, carbon and oxygen) by deducting an amount of helium to keep the sum of the mass fractions equal to unity. At low temperatures $\left(10^{2.7} \mathrm{~K}<T<10^{4.5} \mathrm{~K}\right)$, we use the tables of Ferguson et al. (2005). These tables are available for solar composition, but not for enhanced carbon and oxygen mass fractions compatible with the OPAL tables. In the overlap region between the OPAL and the Ferguson et al. tables $\left(10^{3.75} \mathrm{~K}<T<10^{4.5} \mathrm{~K}\right)$, we give preference to the low-temperature opacity tables, because they take into account the contribution from molecular lines (this contribution is not included in the OPAL tables). For carbon and oxygen enhanced compositions, there are regions of low temperature and density for which opacity values are not available. In these regions, the opacity is generally most sensitive to temperature, and thus we set the opacity to the nearest value that is available at the same temperature. Most of the opacities set this way are below the opacity floor (see the discussion below), so that this deficiency in the tables has a small impact on the light curve evolution. At worst, it may affect the transition between the plateau and the ${ }^{56} \mathrm{Ni}$ dominated part of SN IIP light curves when the photosphere first moves into carbon/oxygen-rich regions.

The Rosseland mean opacity that we obtain from the OPAL and Ferguson et al. tables does not describe all possible sources of opacity needed for simulating SN light curves. As has been argued in previous works (see Karp et al. 1977; Blinnikov 1996; Young 2004; Bersten et al. 2011), the tabulated Rosseland mean opacity calculated for a static medium may underestimate the contribution of the line opacities in the rapidly expanding matter of the exploding star, plus it does not contain possible non-thermal ionization and excitation by gamma-rays. Due to these missing effects, it is common practice to use a so-called opacity floor, effectively imposing a minimum possible value for the opacity. Presently, there is no universally agreed-upon prescription for how to choose this opacity floor for a given composition and velocity. In previous work, different values of the opacity floor were chosen based either on simplified physical arguments (e.g., Shigeyama \& Nomoto 1990) or based on comparisons with results obtained with multi-group or line-transfer codes (e.g., Bersten et al. 2011). For SNe IIP, the values of the opacity floor for the hydrogen-rich envelope and the metal-rich core of the star, as well as the location and shape of the transition between the core/envelope opacities, can strongly influence the shape of the resulting light curve. Qualitatively (as shown in Bersten 2010 and confirmed by our simulations), a lower value of the opacity floor in the envelope of the star increases the plateau luminosity and decreases the duration of the plateau, and vice versa. The luminosity of the plateau and its duration are important observed photometric quantities that are used for statistical studies of SNe IIP (as, e.g., in Anderson et al. 2014). Hence, it is important to keep the uncertainties in the opacity and their propagation into variations of the light curve in mind when comparing modeling results with observations.

In the work of Bersten et al. (2011) on SN IIP light curves, the opacity floor was set to $0.01 \mathrm{~cm}^{2} \mathrm{~g}^{-1}$ for the "envelope" and $0.24 \mathrm{~cm}^{2} \mathrm{~g}^{-1}$ for the "core." Since Bersten et al. (2011) are not specific in defining what constitutes the core and envelope, and because this prescription introduces large opacity discontinuities, we take a different approach in SNEC. We choose the opacity floor to be linearly proportional to metallicity $Z$ at each grid point, and set it to $0.01 \mathrm{~cm}^{2} \mathrm{~g}^{-1}$ for solar composition $(Z=0.02)$ and to $0.24 \mathrm{~cm}^{2} \mathrm{~g}^{-1}$ for a pure metal composition $(Z=1){ }^{6}$ Note that we do not include the opacity floor in the calculation of the optical depth and position of the photosphere (as in Bersten et al. 2011). This is justified by the fact that the opacity floor is used to account for line effects, which have minor influence on the shape of most of the continuum spectrum. However, we note that in the blue part of the spectrum (e.g., in the $U$ and $B$ bands), the continuum may be affected by the numerous lines of iron-group elements (see, e.g., Figure 8 of Kasen \& Woosley 2009).

\subsection{Radioactive ${ }^{56} \mathrm{Ni}$ and Bolometric Luminosity}

Radioactive ${ }^{56} \mathrm{Ni}$ in core-collapse $\mathrm{SNe}$ is synthesized by explosive nuclear burning of intermediate-mass elements during the first seconds of the $\mathrm{SN}$ explosion in the inner regions of the star. It is mixed outward by hydrodynamic instabilities triggered by the shock's propagation through the envelope (see, e.g., Kifonidis et al. 2003, 2006; Wongwathanarat et al. 2015). The gamma-rays, emitted in the ${ }^{56} \mathrm{Ni} \rightarrow{ }^{56} \mathrm{Co} \rightarrow{ }^{56} \mathrm{Fe}$ decay process, diffuse and thermalize, providing an additional source of energy $\epsilon_{\mathrm{Ni}}$ in Equation (2).

The present version of SNEC does not include a nuclear reaction network and the amount and distribution of synthesized ${ }^{56} \mathrm{Ni}$ is provided by the user. While this is a technical limitation that will be removed in future versions of SNEC, one should keep in mind that nucleosynthetic yields are sensitive to (1) how the explosion is launched, (2) where (in mass and spatial coordinate) it is launched, (3) to uncertainties in the structure and composition of the layers in which explosive burning occurs (e.g., Young \& Fryer 2007). Specifying the ${ }^{56} \mathrm{Ni}$ yield by hand removes these uncertainties from our models and has the added benefit of allowing the user complete control of radioactive heating, which can be useful for exploring how it impacts light curves.

For the gamma-rays released in ${ }^{56} \mathrm{Ni}$ and ${ }^{56} \mathrm{Co}$ decay, we follow the gray transfer approximation of Swartz et al. (1995), solving the transfer equation in the form

$$
\frac{d I^{\prime}}{d \tau}=I^{\prime}-X_{\mathrm{Ni}},
$$

where $\tau$ is the optical depth along a given ray, $X_{\mathrm{Ni}}$ is the mass fraction of ${ }^{56} \mathrm{Ni}, I^{\prime}=\left(4 \pi \kappa_{\gamma} / \epsilon_{\mathrm{rad}}\right) I$ and $I$ is the energyintegrated intensity. The effective gamma-ray opacity is assumed to be purely absorptive and independent of energy, $\kappa_{\gamma}=0.06 Y_{e} \mathrm{~cm}^{2} \mathrm{~g}^{-1}$, where $Y_{e}$ is the electron fraction. The time-dependent rate of energy release per gram of radioactive nickel, $\epsilon_{\mathrm{rad}}$, is equal to

$$
\begin{aligned}
\epsilon_{\mathrm{rad}}= & 3.9 \times 10^{10} \exp \left(-t / \tau_{\mathrm{Ni}}\right)+6.78 \times 10^{9}\left[\exp \left(-t / \tau_{\mathrm{Co}}\right)\right. \\
& \left.-\exp \left(-t / \tau_{\mathrm{Ni}}\right)\right] \mathrm{erg} \mathrm{g}^{-1} \mathrm{~s}^{-1},
\end{aligned}
$$

where $\tau_{\mathrm{Ni}}$ and $\tau_{\mathrm{Co}}$ are the mean lifetimes of ${ }^{56} \mathrm{Ni}$ and ${ }^{56} \mathrm{Co}$, equal to 8.8 and 113.6 days, respectively. The local heating rate in each grid point is equal to $\epsilon_{\mathrm{Ni}}=\epsilon_{\mathrm{rad}} d$, where $d$ is the

\footnotetext{
6 For the SNe IIP we study here, we do not find pure-metal regions in our models due to mixing that we impose during the explosion as described in Section 4.1.
} 
deposition function

$$
d=\frac{1}{4 \pi} \oint I^{\prime} d \omega
$$

where $\omega$ is the solid angle.

We do not take into account the energy from positrons released in the radioactive decay of ${ }^{56} \mathrm{Co}$ (which occurs for $19 \%$ of the decays). The total kinetic energy of positrons per ${ }^{56} \mathrm{Co}$ decay is $\sim 0.12 \mathrm{MeV}$ versus $\sim 3.61 \mathrm{MeV}$ emitted via gamma-rays (see Nadyozhin 1994). Therefore, neglecting this contribution constitutes an error of order 3\%-4\% in the overall energetics of the ${ }^{56} \mathrm{Ni}$ decay (Swartz et al. 1995).

Finally, we calculate the observed bolometric luminosity as suggested in Young (2004). It consists of two parts, the luminosity at the photosphere and the luminosity due to the absorption of gamma-rays from ${ }^{56} \mathrm{Ni} /{ }^{56} \mathrm{Co}$ decay above the photosphere

$$
L_{\mathrm{obs}}=L_{\text {photo }}+\int_{m_{\text {photo }}}^{M} S_{\text {dep }}(m) d m .
$$

Here $m_{\text {photo }}$ is the mass coordinate of the photosphere, $M$ is the total mass of the star, $S_{\text {dep }}$ is the energy per gram per second deposited by gamma-rays. The location of the photosphere is defined by the optical depth $\tau=2 / 3$, and $L_{\text {photo }}$ is found from Equation (4) at the photosphere location.

\section{PROGENITOR MODELS WITH VARYING HYDROGEN-RICH ENVELOPE MASSES}

\subsection{Motivation and Overall Strategy}

As a first application of SNEC we investigate the light curves of SNe from massive stars that have lost varying amounts of their hydrogen-rich envelope during their evolution. We use SNEC to explode presupernova models that we generate with the open-source stellar evolution code MESA (release version 6794; Paxton et al. 2011, 2013). The employed MESA input files and final presupernova profiles are available at http:// stellarcollapse.org/SNEC.

Massive stars may lose large fractions of their hydrogen-rich envelopes via steady line-driven winds, via stable or unstable binary mass transfer, or, possibly, through pulsational instabilities and eruptions (see, e.g., Smith 2014 for a recent review). The demographics of SN types combined with initial-massfunction considerations suggest that line-driven winds alone cannot account for the fraction of observed stripped-envelope $\mathrm{SNe}$ (Smith et al. 2011). One of the other avenues of mass loss may be required to partially or completely remove hydrogenrich envelope to account for the fraction of observed $\mathrm{SNe} \mathrm{IIb}$ and $\mathrm{Ib} / \mathrm{c}$. Since virtually all massive stars are born in binaries and up to $70 \%$ of them will interact with their companion (Sana et al. 2012), binary interaction may be the top contender for how massive stars shed their hydrogen-rich envelopes. It is possible that binary interactions (and other massergon:15-loss mechanisms) can result in various degrees of envelope stripping and that there is a broad distribution of hydrogenrich envelope masses at the presupernova stage of stars of any given zero-age main sequence (ZAMS) mass.

Our goal here is to study the effects of substantial mass loss during massive star evolution on presupernova structure and the resulting $\mathrm{SN}$ light curve. We assume that the mass is lost rapidly (e.g., in an unstable mass transfer event or through some instability), but sufficiently slowly that the star can re-

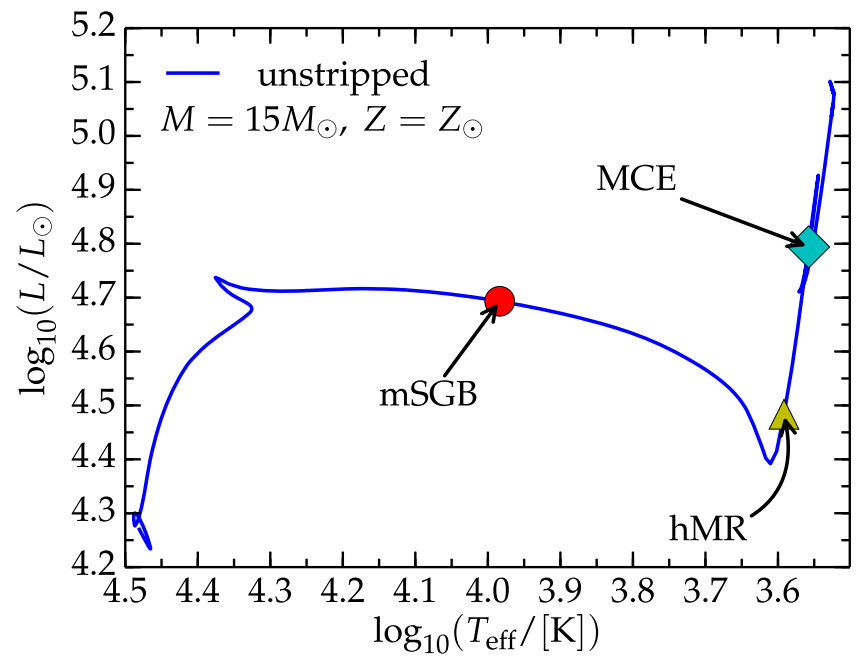

Figure 1. Evolutionary track on the Hertzsprung-Russell diagram for the unstripped reference $15-M_{\odot}$ (at ZAMS) star computed with MESA. Each mark corresponds to a post-MS evolutionary stage at which we strip: mSGB (red dot) stands for "middle of the subgiant branch" defined via $T_{\mathrm{eff}}=10^{4} \mathrm{~K}$; hMR (yellow triangle) stands for "half maximum radius," and MCE (cyan rhombus) stands for "maximum extent of convective envelope." While these three stripping points are separated only by $\sim 10^{4}$ years, they sample an interesting range of structure and an order of magnitude in envelope extent. See Table 1 for more quantitative information on the stripping points. Each stripped model corresponds to the reference model up to its stripping point and is stripped only once.

adjust to a new equilibrium after the mass loss event. Rather than attempting to self-consistently simulate various highly uncertain mass loss mechanisms, we instead conduct a controlled experiment in stellar astrophysics by systematically stripping material from the envelope of a fiducial $M_{\text {ZAMS }}=15 M_{\odot}$ star at different points of its evolution. We note that Bayless et al. (2015) carried out a similar study of the effects of mass stripping on SN II light curves. However, they considered a $23-M_{\odot}$ progenitor model and stripped it only at the presupernova stage.

Figure 1 shows the evolutionary track on the HertzsprungRussel diagram for the $15-M_{\odot}$ reference model. Rapid mass loss is most likely to occur in the post-MS evolution because the envelope expands and becomes only weakly bound to the compact core. When precisely the mass loss event occurs and how much mass is lost will depend on the process causing mass loss and possibly widely varying parameters such as the details of binary configuration. In order to account for our ignorance of the details of the mass loss event, we consider three points (indicated by symbols in Figure 1; see also Table 1) in the postMS evolution of our reference model that probe different envelope structures and span envelope radii from $\sim 80 R_{\odot}$ to $\sim 640 R_{\odot}$.

1. $m S G B$ series: these models are stripped at $T_{\text {eff }}=10^{4} \mathrm{~K}$, which marks the middle of the subgiant branch (mSGB). At this point, the star's envelope has expanded to a radius of $79.8 R_{\odot}$. Hydrogen is burning in a shell and the $\sim 5 M_{\odot}$ helium-rich core is inert. The envelope is still mostly radiative with a convective layer at mass coordinates 5.5-6.5 $M_{\odot}$.

2. $h M R$ series: these models are stripped when the radius first surpasses the half-maximum radius (hMR) of the reference model, $R \sim 375 R_{\odot}$. At this point, the envelope region outside a mass coordinate of $m \sim 9.5 M_{\odot}$ is 
Table 1

Summary of Stripping Points

\begin{tabular}{|c|c|c|c|c|c|c|}
\hline Series Name & Stripping Criterion & Age (Myr) & $R\left(R_{\odot}\right)$ & $M_{\mathrm{H}}\left(M_{\odot}\right)$ & $M_{\mathrm{He}}\left(M_{\odot}\right)$ & $\max \{\Delta M\}$ \\
\hline Middle SGB (mSGB) & $T_{\text {eff }}=10^{4} \mathrm{~K}$ & 13.0263 & 379.8 & 10.67 & 3.81 & 7 \\
\hline Half Maximum Radius (hMR) & $R \gtrsim 375 R_{\odot}$ & 13.0305 & 381.6 & 10.62 & 3.87 & 7 \\
\hline $\begin{array}{l}\text { Maximum Extent } \\
\text { of the Convective Envelope (MCE) }\end{array}$ & $\begin{array}{c}X_{c}=0 \text { and } \\
\left(v_{\text {conv }}^{\text {surf }}-v_{\text {conv }}^{\text {env }}\right) / v_{\text {conv }}^{\text {surf }} \leqslant 0.1\end{array}$ & 13.0310 & 638.1 & 10.61 & 3.88 & 7 \\
\hline
\end{tabular}

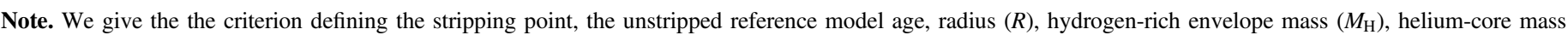

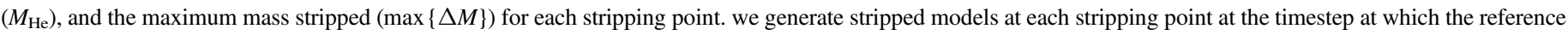

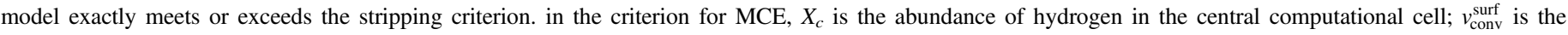

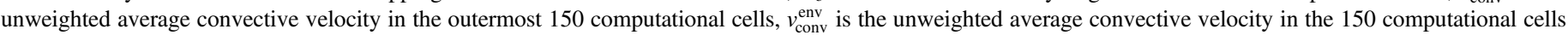
above the outermost lower boundary of a convective region. If these two differ by less than $10 \%$, the envelope has roughly reached its maximum extent.

convective while deeper layers are radiative. There is hydrogen shell burning and a small core helium burning region.

3. MCE series: these models are stripped when the maximum radial extent of the convective envelope is reached (MCE, at a stellar radius of $R \sim 638 R_{\odot}$ ). The outer $\sim 8 M_{\odot}$ of the star are fully convective at this point. There is hydrogen shell burning and a small core helium burning region.

In each series of models, we strip in units of $1 M_{\odot}$, but stop before we reach the hydrogen shell burning region and keep at least $1 M_{\odot}$ of the radiative layer that surrounds it. Note that if we considered Roche-lobe overflow in a binary system as the mechanism for mass loss, then only the outer convective layers could be lost in an unstable Roch-lobe overflow event, but stripping of radiative layers would not occur dynamically (cf. Hilditch 2001).

The time spanned by mSGB-hMR-MCE is only of order $10^{4}$ years, which is small compared to the full lifetime of the unstripped reference model $\left(\sim 14.13 \times 10^{6}\right.$ years $)$. It is, however, very large compared to the dynamical time and the thermal Kelvin-Helmholtz time of the star. The latter is $t_{\mathrm{KH}} \sim 3 / 4 G M^{2} /(R L)$ (Kippenhahn et al. 2012), which is $\sim 1250$ years, $\sim 425$ years, and $\sim 125$ years at mSGB, hMR, and MCE, respectively. After stripping, about $\sim 10^{6}$ years of evolution are left until core collapse.

\subsection{MESA Simulations}

We employ MESA release version 6794 (Paxton et al. 2011, 2013) and assume solar metallicity $Z_{\odot}=0.019$. We use the Ledoux criterion for convection and follow Sukhbold \& Woosley (2014), who set the mixing length parameter $\alpha_{\mathrm{mlt}}=2.0$, the overshooting parameter $f_{\mathrm{ov}}=0.025$, and semiconvection efficiency $\alpha_{\mathrm{sc}}=0.01$, and do not consider thermohaline mixing. We use the wind mass loss prescription of Vink et al. $(2000,2001)$ for the hot MS phase and that of de Jager et al. (1988) for the cool giant phase, both with $\eta=1.0$. We limit MESA's timestep by enforcing fractional changes in structure and thermodynamics variables of less than $10^{-3}$ per timestep (varcontrol_target $=10^{-3}$ ) and also use a customized timestep control that enforces a timestep that is always smaller than the model's Kelvin-Helmholtz time. We use MESA's standard setting for rezoning, mesh_delta_coeff $=1.0$, and mesh_delta_coeff_for_hight $=1.5$, which coarsens the resolution at $T \gtrsim 10^{9} \mathrm{~K}$ and, thus, in the core region, where we do not currently trust MESA results (see below). These standard resolution setting provide a sufficiently resolved envelope for our $\mathrm{SNEC}$ explosion and light curve simulations.

For simplicity and speed of execution, we simulate all models with MESA's default 21-isotope nuclear reaction network approx21 until the onset of core collapse, which is commonly defined as the point when the infall velocity reaches $1000 \mathrm{~km} \mathrm{~s}^{-1}$. We note that much larger (100-1000 isotope) networks are needed for an accurate treatment of late oxygen burning and silicon burning and of the pre-collapse neutronization in the degenerate core (e.g., Sukhbold \& Woosley 2014; W. D. Arnett 2015, private communication). Since the treatment of these late burning stages has a large effect on the core region out to the carbon-oxygen-helium interface (Sukhbold \& Woosley 2014), the core structure of our MESA models is not reliable. Core collapse and postbounce supernova simulations that focus on the explosion mechanism suggest that the structure in the inner 1.4-2.5 $M_{\odot}$ may determine if neutrino-driven explosions fail or succeed (O'Connor \& Ott 2011; Ugliano et al. 2012; Ertl et al. 2015). However, for our explosion study with SNEC, details of the core structure are not essential, since the outer regions and the hydrogen-rich envelope determine the light curve. We also artificially launch explosions and introduce ${ }^{56} \mathrm{Ni}$ by hand (see Section 2).

\subsection{Stripping Procedure}

We first evolve copies of the unstripped reference model to the onset of core collapse, and to the three stripping points: mSGB, hMR, and MCE (see Table 1 and Figure 1). At these stripping points, we then restart and use MESA's module adjust_mass to "instantaneously" remove the specified amount of mass. The new smaller value of the total mass is reached using 80 MESA "pseudo-evolution" steps (i.e., the structure is evolved using timesteps, but the time coordinate is held constant). During each step, MESA removes the largest amount of mass from the envelope that it can while still finding a hydrostatic solution to the structure equations. In most cases, $\sim 75$ are sufficient to reach the desired mass and the last $\sim 5$ have mass loss set to zero. Both during and at the end of the pseudo-evolution, the structure is always in global hydrostatic equilibrium, therefore, when the regular evolution resumes, no readjustment occurs.

We strip mass in $1 M_{\odot}$ steps and continue the evolution of each model to the onset of core collapse. We refer to the unstripped reference model simply as "unstripped," and name the stripped models according to [stripping point]_[number of $M_{\odot}$ stripped]M. For example, "hMR_5M" stands for a model that had $5 M_{\odot}$ stripped at hMR. 
Table 2

Summary of the Presupernova Structure of the MESA Models

\begin{tabular}{|c|c|c|c|c|c|c|c|c|c|c|c|}
\hline Model & $M_{\text {pre- SN }}\left(M_{\odot}\right)$ & $M_{\mathrm{H}}\left(M_{\odot}\right)$ & $M_{\mathrm{He}}\left(M_{\odot}\right)$ & $M_{\mathrm{CO}}\left(M_{\odot}\right)$ & $M_{\mathrm{Fe}}\left(M_{\odot}\right)$ & $\left|E_{\mathrm{b}}\right|(\mathrm{B})$ & $\xi_{2.5}^{\text {pre- SN }}$ & $R\left(R_{\odot}\right)$ & $L\left(L_{\odot}\right)$ & $T_{\text {eff }}\left(10^{3} \mathrm{~K}\right)$ & $t_{\mathrm{SB}}(\mathrm{h})$ \\
\hline unstripped & 12.28 & $\lesssim 7.18$ & 5.10 & 3.27 & 1.51 & 0.641 & 0.103 & 1039 & 120309 & 3.337 & 48.52 \\
\hline mSGB_1M & 11.27 & $\lesssim 6.18$ & 5.09 & 3.28 & 1.49 & 0.697 & 0.125 & 1031 & 121084 & 3.355 & 45.71 \\
\hline mSGB_2M & 10.25 & $\lesssim 5.16$ & 5.09 & 3.26 & 1.49 & 0.617 & 0.142 & 1013 & 119370 & 3.373 & 42.79 \\
\hline mSGB_3M & 9.17 & $\lesssim 4.06$ & 5.11 & 3.27 & 1.49 & 0.586 & 0.127 & 991 & 121536 & 3.425 & 38.74 \\
\hline mSGB_4M & 7.87 & $\lesssim 2.67$ & 5.20 & 3.32 & 1.58 & 0.749 & 0.138 & 932 & 122270 & 3.537 & 31.99 \\
\hline mSGB_5M & 6.82 & $\lesssim 1.61$ & 5.21 & 3.33 & 1.56 & 0.734 & 0.171 & 828 & 122984 & 3.759 & 25.11 \\
\hline mSGB_6M & 5.94 & $\lesssim 0.74$ & 5.20 & 3.32 & 1.54 & 0.650 & 0.114 & 663 & 123258 & 4.204 & 16.75 \\
\hline mSGB_7M & 5.59 & $\lesssim 0.38$ & 5.21 & 3.33 & 1.50 & 0.625 & 0.089 & 555 & 118763 & 4.553 & 12.25 \\
\hline hMR_1M & 11.27 & $\lesssim 6.18$ & 5.09 & 3.28 & 1.49 & 0.697 & 0.125 & 1031 & 121084 & 3.355 & 45.71 \\
\hline hMR_2M & 10.25 & $\lesssim 5.16$ & 5.09 & 3.26 & 1.49 & 0.617 & 0.142 & 1013 & 119370 & 3.373 & 42.79 \\
\hline hMR_3M & 9.17 & $\lesssim 4.06$ & 5.11 & 3.27 & 1.49 & 0.586 & 0.127 & 991 & 121536 & 3.425 & 38.74 \\
\hline hMR_4M & 7.87 & $\lesssim 2.67$ & 5.20 & 3.32 & 1.58 & 0.749 & 0.138 & 932 & 122270 & 3.537 & 31.99 \\
\hline hMR_5M & 6.87 & $\lesssim 1.68$ & 5.19 & 3.32 & 1.53 & 0.658 & 0.118 & 843 & 122179 & 3.719 & 25.58 \\
\hline hMR_6M & 5.96 & $\lesssim 0.77$ & 5.18 & 3.31 & 1.58 & 0.709 & 0.122 & 676 & 122065 & 4.153 & 17.19 \\
\hline hMR_7M & 5.52 & $\lesssim 0.32$ & 5.21 & 3.30 & 1.60 & 0.706 & 0.110 & 551 & 122645 & 4.604 & 11.83 \\
\hline MCE_1M & 11.27 & $\lesssim 6.17$ & 5.10 & 3.27 & 1.58 & 0.765 & 0.102 & 1032 & 118857 & 3.339 & 45.62 \\
\hline MCE_2M & 10.25 & $\lesssim 5.16$ & 5.09 & 3.27 & 1.54 & 0.644 & 0.134 & 1016 & 120982 & 3.379 & 42.79 \\
\hline MCE_3M & 9.17 & $\lesssim 4.06$ & 5.11 & 3.27 & 1.53 & 0.696 & 0.159 & 989 & 119197 & 3.413 & 38.65 \\
\hline MCE_4M & 7.88 & $\lesssim 2.69$ & 5.19 & 3.32 & 1.58 & 0.592 & 0.130 & 932 & 122808 & 3.541 & 32.36 \\
\hline MCE_5M & 6.87 & $\lesssim 1.68$ & 5.19 & 3.32 & 1.52 & 0.708 & 0.131 & 843 & 121709 & 3.715 & 25.55 \\
\hline MCE_6M & 5.96 & $\lesssim 0.78$ & 5.18 & 3.31 & 1.55 & 0.694 & 0.153 & 675 & 122791 & 4.162 & 17.20 \\
\hline MCE_7M & 5.52 & $\lesssim 0.31$ & 5.21 & 3.31 & 1.56 & 0.718 & 0.123 & 552 & 122631 & 4.602 & 11.80 \\
\hline
\end{tabular}

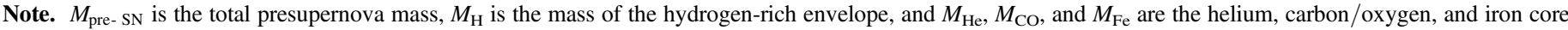

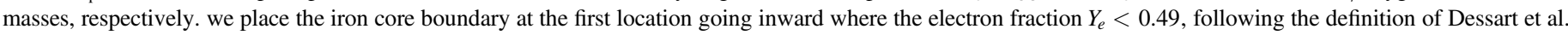

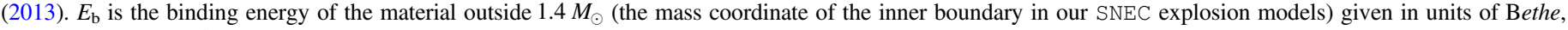

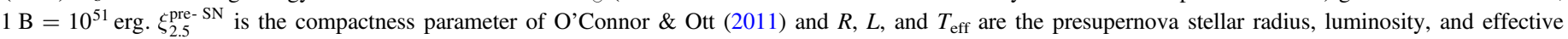
temperature. $t_{\mathrm{SB}}$ is the time of shock breakout in hours after the onset of the thermal bomb.

\subsection{Resulting Presupernova Structure}

We summarize the presupernova structure of our model set in Table 2 and Figure 2. The unstripped reference model reaches the presupernova stage as a $12.28 M_{\odot}$ RSG with a hydrogen-rich envelope mass of $M_{\mathrm{H}} \sim 7.18 M_{\odot}$. The stripped models have envelopes of systematically lower mass, approximately proportional to the amount of mass removed. While $M_{\mathrm{H}}$ varies by more than a factor of seven $\left(M_{\mathrm{H}} \sim 0.31-0.38\right.$ for the most stripped models) within a model series, the final stellar radius varies only by a factor of $\sim 2$. Hence, the envelopes become increasingly dilute (lower-density) with increasing stripping. Following the $T_{\text {eff }}$ criterion of Georgy (2012), our models with less than $6 M_{\odot}$ stripped die as RSGs while models from which we strip $6 M_{\odot}$ or $7 M_{\odot}$ die as yellow supergiants. It is apparent from Figure 2 that the choice of stripping point has almost negligible influence on the final mass and structure of the remaining hydrogen-rich envelope. Differences in $M_{\mathrm{H}}$ and radius $R$ are generally $\lesssim 5 \%$ between models from different series that had the same amount of mass removed. An exception are the most extreme _M7 cases $\left(\begin{array}{lll}7 & M_{\odot} & \text { of }\end{array}\right.$ hydrogen-rich material stripped) that show a $\sim 20 \%$ variation in their final $M_{\mathrm{H}}$ from $0.31 M_{\odot}$ in model MCE_7M, $0.32 M_{\odot}$ in model hMR_7M, to $0.38 M_{\odot}$ in model mSGB_7M. The envelope in the latter model temporarily becomes compact when helium ignites in the core, which leads to less wind mass loss after stripping. The radii of all_7M models at core collapse are nearly identical $\left(\sim 550 R_{\odot}\right)$.

The iron core mass $\left(\sim 1.5-1.6 M_{\odot}\right)$ and density profile is nearly identical in all models. They reach core collapse at central densities in the range $0.93-1.48 \times 10^{10} \mathrm{~g} \mathrm{~cm}^{-3}$. Since the electrons in the iron core are relativistically degenerate and the core specific entropy and electron fraction are roughly the same in all models, the iron core structure is very similar throughout the model series. More interesting are the large variations in the density profiles in the silicon and oxygen/ carbon layers above the iron core, between $\sim 1.5 M_{\odot}$ and $\sim 3.3 M_{\odot}$, as shown in Figure 2. The presupernova structure in these layers appears to be very sensitive to both the amount of envelope mass stripped and the stripping point in the evolution. However, there are no identifiable trends that could be linked to amount of mass stripped and stripping point. Sukhbold \& Woosley (2014) pointed out that the structure in these layers is sensitive to the treatment of (1) nuclear reactions and weak interactions (neutrino cooling, neutronization) and (2) mixing and overshooting. Both (1) and (2), in turn, influence the number and extent of convective shell burning episodes/ regions in the silicon and carbon/oxygen layers. Our results indicate that variations in the time and amount of mass loss can also influence this part of presupernova stellar structure. The density distribution in the affected regions determines the compactness parameter of O’Connor \& Ott (2011),

$$
\xi_{M} \stackrel{\text { def }}{=} \frac{M / M_{\odot}}{R(M) / 1000 \mathrm{~km}},
$$

with the commonly adopted reference value $M=2.5 M_{\odot}$. Multiple studies (e.g., O'Connor \& Ott 2011; Ugliano et al. 2012; Ertl et al. 2015) have demonstrated that the compactness parameter $\xi_{2.5}$ is a useful quantity to (at a roughly 


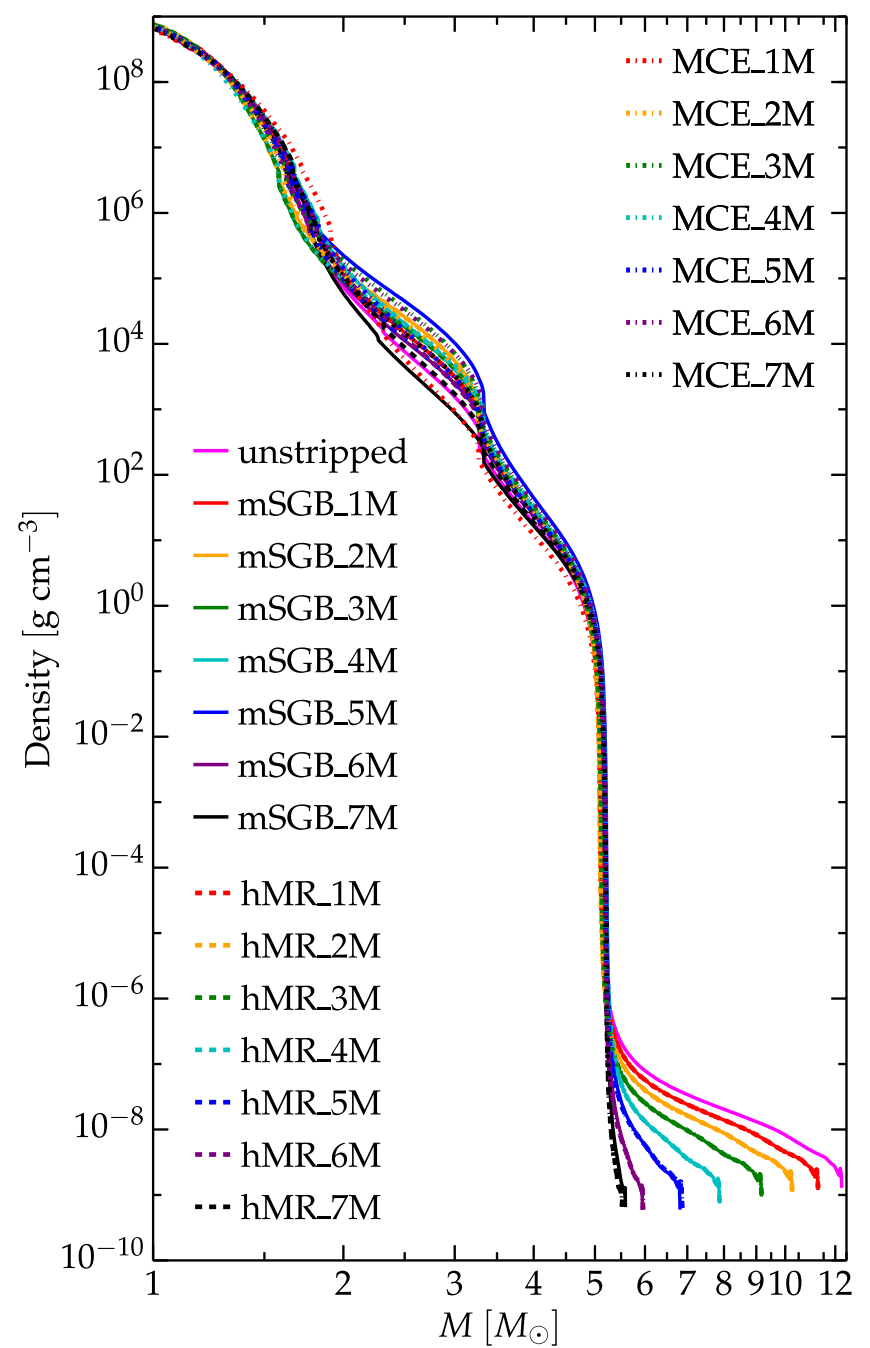

Figure 2. Density profiles as a function of enclosed mass at the onset of core collapse of the entire set of MESA presupernova models computed in this study. The solid curves include the unstripped reference model and the models stripped at mSGB, dashed curves show models stripped at hMR, and dotdashed curves represent models stripped at MCE (cf. Figure 1 and the text for a discussion of these stripping points). More stripped models have more tenuous envelopes, but the time of stripping has negligible influence on the envelope structure. However, both time of stripping and the amount of mass stripped influence the structure of the silicon and carbon/oxygen layers around 1.5-3.3 $M_{\odot}$ in a complex and not obviously systematic way. The structure in this region may determine the outcome of core collapse (cf. O'Connor \& Ott 2011; Ugliano et al. 2012; Ertl et al. 2015).

"first order" level) judge whether a given star is more likely to explode in a supernova or collapse to a black hole without explosion. Hence, the dependence of $\xi_{2.5}$ on mass loss (both rapid and due to winds) deserves further investigation in future work with a version of MESA with a much larger nuclear reaction network and a more reliable treatment of the final stages of stellar evolution.

\section{LIGHT CURVES}

We next present our study of the light curves from exploding the stripped MESA models described above. We begin by summarizing our basic setup in Section 4.1. We then present the light curve of an unstripped model in Section 4.2 that will serve as a reference for the subsequent discussion of stripped models. We also include a discussion of how details such as the mixing, the nickel distribution, and how the explosion is initiated impact the light curves in Section 4.3. Finally, we present our main study of the light curves from models with varying levels of stripping in Section 4.4.

\subsection{Explosion and Light Curve Setup}

In all explosion models, we excise the inner $1.4 M_{\odot}$, assuming that this part collapsed and formed a neutron star. We then map (via linear interpolation) the hydrodynamic and compositional variables from MESA to a 1000 cell grid in SNEC. We choose the grid spacing so that resolution is concentrated in the interior, where the thermal bomb is placed, and near the surface, where the photosphere is initially located. In our fiducial resolution calculations, the innermost cell has a mass of $\Delta m=6.5 \times 10^{-3} M_{\odot}$ and the surface cell has a mass of $\Delta m=6.5 \times 10^{-5} M_{\odot}$. The lowest resolution in our fiducial setup is $\Delta m=0.065 M_{\odot}$ at mass coordinates between $\sim 2.5$ and $\sim 5 M_{\odot}$ (at around grid cell 100). The mass of cells between the innermost cell and the coarsest cell changes according to geometric progression with a fixed ratio between two consecutive cells $>1$. Between the coarsest cell and the surface cells we refine by geometric progression with a fixed ratio between two consecutive cells $<1$. Examples of the grids may be found in Appendix A. The release version of SNEC contains a routine to generate a variety of grid setups.

The explosion is initiated by applying a "thermal bomb" to the innermost region of the model, just above the mass cut in a way similar to previous work (e.g., Aufderheide et al. 1991; Blinnikov \& Bartunov 1993; Bersten et al. 2011). The energy of the bomb is added to the right-hand side of Equation (2) during a chosen time interval and in a range of mass from the inner boundary, both exponentially attenuated. For our fiducial model, summarized in Section 4.2, the bomb is spread over $0.02 M_{\odot}$ and injected in $1 \mathrm{~s}$. The thermal bomb mechanism, implemented this way, typically gives a few percent more energy to the system than dialed-in, due to the smooth exponential attenuation. This small excess in energy is recorded and accounted for in SNEC's global energy balance. We find that SNEC conserves total energy to better than $1 \%$ in a fullphysics model followed to 150 days past explosion.

An alternative way of phenomenologically modeling an SN explosion is the "piston mechanism," which has been used, for example, in the work by Eastman et al. (1994), Utrobin (2007), Kasen \& Woosley (2009), and Dessart et al. (2013). For the same amount of injected energy, the thermal bomb and piston mechanism give nearly identical light curves, as was discussed in Bersten et al. (2011) and confirmed by our own simulations. However, when a reaction network is included, piston and thermal bomb may result in different nucleosynthetic yields (Young \& Fryer 2007). We have implemented a piston in $\mathrm{SNEC}$, but do not use it in this work, since the thermal bomb makes it easier to control the energy of the explosion.

It has long been realized in SN light curve modeling that sharp gradients in the composition profiles of the progenitors may result in artificial light curve features that are not observed in real SNe. For example, Utrobin (2007) points out a pronounced bump at the end of the plateau, followed by an abrupt decrease of the bolometric luminosity for a model with unmixed chemical composition (see their Figure 16). Two- and three-dimensional simulations of shock propagation in corecollapse SN explosions (e.g., Kifonidis et al. 2003, 2006; 


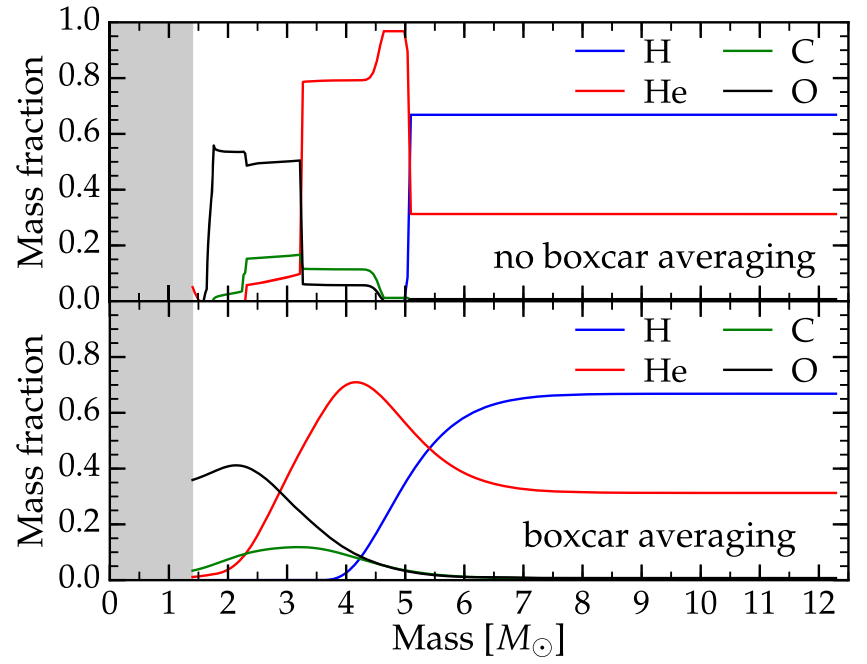

Figure 3. Mass fractions of the key elements hydrogen, helium, oxygen, and carbon in the unstripped reference model before (upper panel) and after (lower panel) boxcar smoothing. Note that the smoothed profile here is shown for a grid with uniform spacing in mass. In our production setup that uses a nonuniform grid with higher resolution in the innermost and outermost regions, small jumps in the composition profiles remain, but have no influence on the light curve. We excise the mass inside the shaded regions before launching the explosion.

Wongwathanarat et al. 2015) show that effective mixing occurs during the shock propagation through the progenitor due to the Rayleigh-Taylor and Richtmyer-Meshkov instabilities. In this process, hydrogen and helium get mixed into the inner layers, while metal-rich clumps, and, in particular, ${ }^{56} \mathrm{Ni}$, may penetrate outwards up to $\sim 3000 \mathrm{~km} \mathrm{~s}^{-1}$ and more in the velocity profile. Lacking a physical mechanism for the mixing in our onedimensional code, we apply an artificial "boxcar" averaging, as used, for example, in Kasen \& Woosley (2009) and Dessart et al. $(2012,2013)$. We run a boxcar with a width of $0.4 M_{\odot}$ through the model four times until we obtain a smooth profile (details of this procedure are available in the SNEC notes document on the SNEC website). As an example, Figure 3 depicts the non-mixed (top panel) and mixed (bottom panel) mass fractions of hydrogen, helium, oxygen, and carbon in the unstripped reference model.

Finally, we assume a fixed amount of ${ }^{56} \mathrm{Ni}$ of $0.05 M_{\odot}$ in all our models, which is roughly the average amount deduced for SNe IIP (which have a range of $\sim 0.01-0.1 M_{\odot}$, see Kasen \& Woosley 2009; Smartt et al. 2009). We distribute it uniformly in the interval between the excised mass of $1.4 M_{\odot}$ and some chosen mass coordinate $\left(5 M_{\odot}\right.$ for the reference runs, which is near the edge of the helium core) at the expense of other elements before smoothening the composition.

\subsection{Fiducial Light Curve of the Unstripped Reference Progenitor Model}

Figure 4 shows the light curve of the unstripped reference model. The explosion was initiated at $t=0$ with a thermal bomb resulting in an asymptotic kinetic energy of $E=10^{51} \mathrm{erg}$ (as described in Section 4.1). We added $M_{\mathrm{Ni}}=0.05 M_{\odot}$, mixed up to a mass coordinate of $5 M_{\odot}$ (but not into the hydrogen envelope, though boxcar smoothing introduces some nickel into the hydrogen-rich region.). These are the fiducial explosion parameter choices used throughout this study.

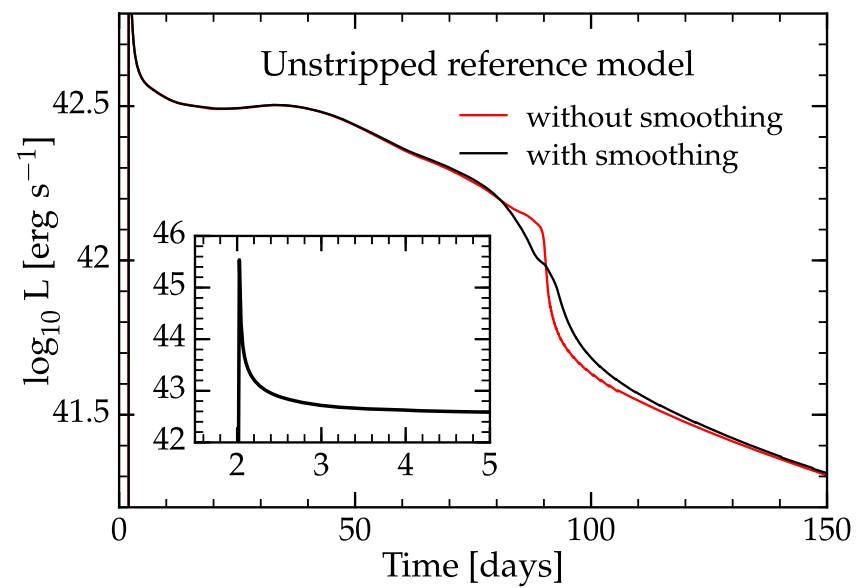

Figure 4. Bolometric light curve of the unstripped reference model. Time is given relative to the onset of the thermal bomb driving the explosion. Shock breakout occurs at day 2.03 through the reference model's surface at $\sim 1039 R_{\odot}$. The black graph is the fiducial light curve obtained with our standard parameter choices, including boxcar smoothing as an approximation of mixing during the explosion (cf. Figure 3 and Section 4.1). The red graph represents the unmixed case with steep compositional gradients (top panel of Figure 3). The inset plot shows shock breakout and the very early light curve. We note that during shock breakout the photosphere is located in the outermost cell of SNEC's grid and spatially poorly resolved. Thus the light curve predicted by SNEC around the time of shock breakout is likely not reliable (cf. Ensman \& Burrows 1992).

The light curve of the unstripped reference model shows all the traditional hallmarks of a typical SNe IIP (e.g., Falk \& Arnett 1977; Eastman et al. 1994; Filippenko 1997). Shock breakout occurs when the optical depth is less than $\sim c / v$ at a time of 2.03 days after the onset of explosion. The bolometric luminosity peaks at $L=3.4 \times 10^{45} \mathrm{erg} \mathrm{s}^{-1}$ with an effective temperature of $T_{\text {eff }}=1.7 \times 10^{5} \mathrm{~K}$. The subsequent cooling phase (discussed in detail in Nakar \& Sari 2010) lasts for $\sim 19$ days. At this point, the ejecta have expanded and cooled so much that hydrogen recombination sets in (starting already at $T \sim 7500 \mathrm{~K}$ ) and powers the plateau phase with very slowly decreasing effective temperature that varies from $\sim 6000 \mathrm{~K}$ at $\sim 35$ days down to $\sim 5000 \mathrm{~K}$ at $\sim 90$ days. The recombination wave and, consequently, the photosphere moves inward in mass coordinate, but due to the overall expansion stays at roughly constant radius, resulting in a relatively small variation in luminosity during the recombination phase of the plateau from day $\sim 19$ to day $\sim 80-90$ (this phase is investigated analytically in Goldfriend et al. 2014). The slow decline that is apparent in the plateau phase shown in Figure 4 occurs because of the combined effects of the photosphere receding slightly in radius and the effective temperature slowly decreasing (e.g., Eastman et al. 1994; Woosley 1988).

The plateau ends when the photosphere reaches the helium core. Helium recombines at $T \gtrsim 10^{4} \mathrm{~K}$ whereas the photospheric temperature is $T \sim 5000 \mathrm{~K}$, recombination accelerates dramatically, and both the radius of the photosphere and the luminosity decrease rapidly. Note that it is common in the SN IIP theoretical light curve literature to define the plateau duration as the time from shock breakout to the drop when the photosphere reaches the helium core (Popov 1993; Kasen \& Woosley 2009). We adopt this definition of plateau duration in this paper. The small "knee" or "bump" feature in the drop of the fiducial light curve around day 90 in Figure 4 is due to the additional luminosity input from radioactive ${ }^{56} \mathrm{Co}$ (from the 


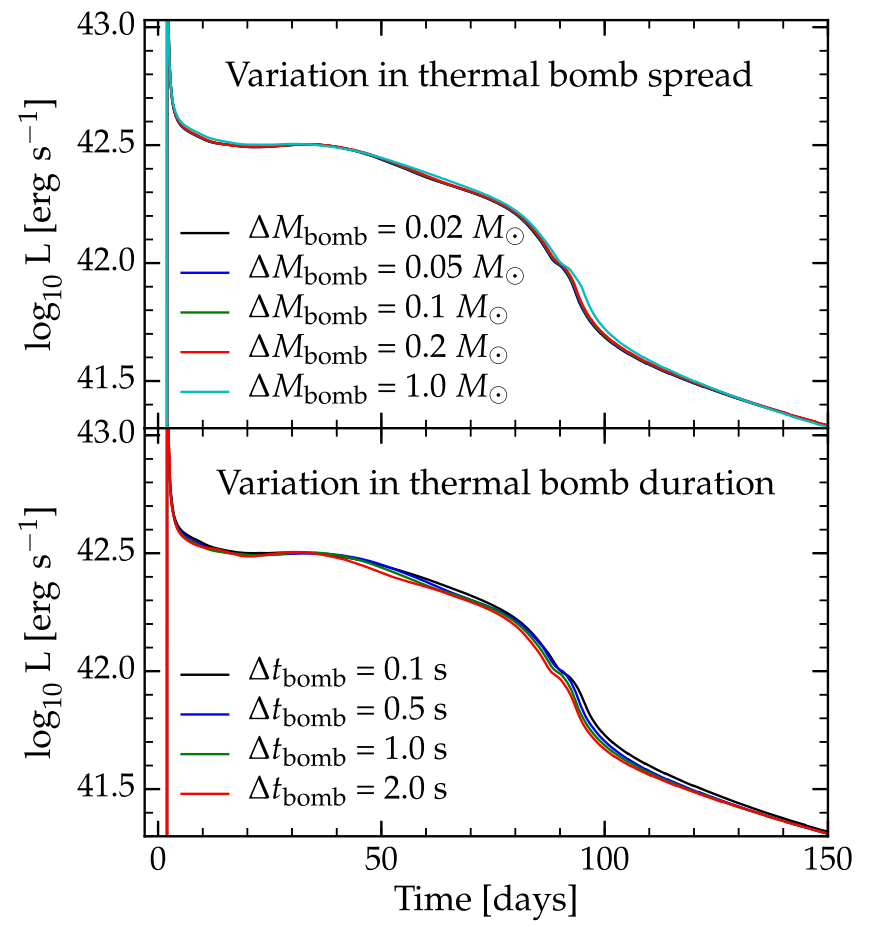

Figure 5. Bolometric light curves of the unstripped reference progenitor computed with different mass range over which the thermal bomb is spread (top panel) and different durations of the thermal bomb (bottom panel). All other parameters are those laid out in Section 4.1. Time is relative to the onset of the thermal bomb.

${ }^{56} \mathrm{Ni} \rightarrow{ }^{56} \mathrm{Co} \rightarrow{ }^{56} \mathrm{Fe}$ decay chain) that is uncovered as the photosphere sweeps through the helium core throughout which ${ }^{56} \mathrm{Ni}$ was mixed initially. This feature is sensitive to the degree and implementation of mixing and is unlikely to be robust (see next Section 4.3). Finally, the tail of the light curve, after day $\sim 100$, is powered exclusively by the radioactive decay of ${ }^{56} \mathrm{Co}$.

\subsection{Sensitivity of the Fiducial Light Curve to Mixing, Thermal Bomb Parameters, and Nickel Distribution}

The red curve in Figure 4 highlights the effect of steep compositional gradients on the light curve in comparison with the result obtained with compositional smoothing (black graph; "boxcar averaging"; Section 4.1) that we use to mimic multidimensional mixing during the explosion. If exploded without smoothing, hydrogen-rich material transitions discontinuously to helium-rich material (cf. Figure 3), which leads to more rapid recombination, a more abrupt drop of the photosphere radius, and a steeper decline of the luminosity. Although observations of most $\mathrm{SNe}$ do not generally reveal such abrupt drops, some subclasses of $\mathrm{SNe}$ may have rapidly dropping photospheric velocities as discussed by Piro \& Morozova (2014) because of this same effect of rapid helium recombination. For all other light curves presented in this paper, we use the smoothed composition profiles.

In Figure 5, we explore the sensitivity of the fiducial light curve to (top panel) variations in the amount of mass over which the thermal bomb is spread and (bottom panel) variations in the duration over which the energy is injected. While the details of the energy injection will depend on the actual physical explosion mechanism (e.g., Bethe 1990; Janka 2012), it is reassuring that the light curve is fairly insensitive to both mass spread and duration of energy injection. We remind the

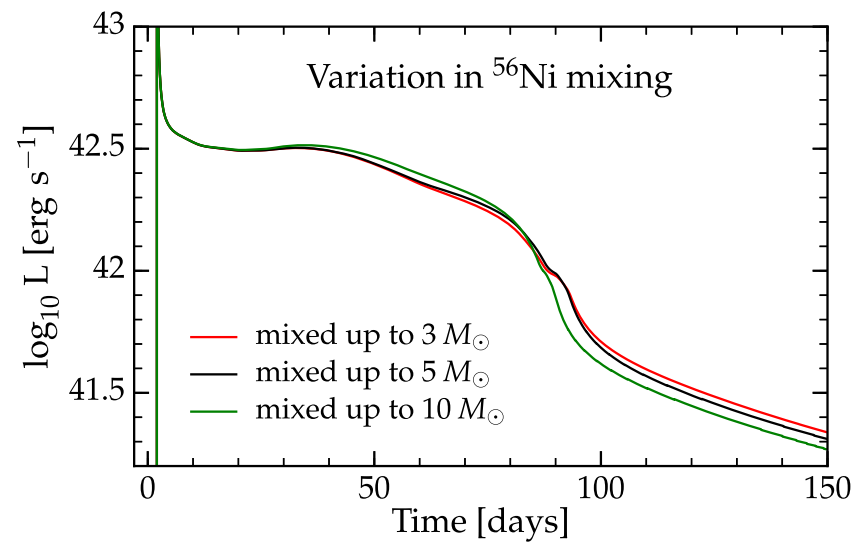

Figure 6. Bolometric light curves for the unstripped reference progenitor model with different degree and extent of initial ${ }^{56} \mathrm{Ni}$ mixing and all other parameters as laid out in Section 4.1. The helium core mass is $5.1 M_{\odot}$ and if ${ }^{56} \mathrm{Ni}$ is mixed smoothly into the hydrogen-rich envelope (green graph), then the light curve's "knee" feature visible when the photosphere drops into the helium core disappears.

reader that for the light curves shown in Sections 4.2 and 4.4, the thermal bomb is spread over $0.02 M_{\odot}$ and its duration is $1 \mathrm{~s}$ (in self-consistent multi-dimensional core-collapse SN simulations most of the energy injection appears to occur within 1-2 s; e.g., Bruenn et al. 2013).

Finally, Figure 6 shows the dependence on ${ }^{56} \mathrm{Ni}$ mixing. The overall effect of ${ }^{56} \mathrm{Ni}$ mixing is modest. The general trend is that with the initial mixing of ${ }^{56} \mathrm{Ni}$ to increasing mass coordinates, the contribution to the luminosity of the ${ }^{56} \mathrm{Ni}$ $\rightarrow^{56} \mathrm{Co} \rightarrow{ }^{56} \mathrm{Fe}$ decay chain becomes more prominent. At later times, the luminosity due to radioactive decay becomes smaller with increasing mixing due to the fact that more gamma-rays escape from the model without being absorbed (as also described by Young 2004; Utrobin 2007; Bersten et al. 2011). Note that if ${ }^{56} \mathrm{Ni}$ is mixed far into the hydrogenrich envelope, the "knee" feature at the end of the plateau disappears. This is consistent with the findings of Kasen \& Woosley (2009), who mixed ${ }^{56} \mathrm{Ni}$ into the hydrogen-rich envelope in their models.

\subsection{Light Curves as a Function of Mass Stripping}

Figure 2 demonstrates that the structure of the hydrogen-rich envelope and of the outer helium core are essentially independent of the point at which we remove mass for the set of stripping points we choose in this study (cf. Section 3.4). This suggests that the resulting light curves should be independent of the stripping point and we check this assertion later in this section. Here, we operate under the assumption that it is true and focus our discussion on the model series stripped at the mSGB.

In the top panel of Figure 7, we show bolometric mSGB series light curves obtained for a final ejecta kinetic energy of $E_{\text {kin }}=10^{51} \mathrm{erg}$ and $M_{\mathrm{Ni}}=0.05 M_{\odot}$ (and all other explosion parameters as specified in Section 4.1). The early, shock breakout and cooling part of the light curves is shown in Figure 8. Shock breakout itself is not well resolved (i.e., the photosphere is in the outermost grid cell) and thus the light curves in this phase are unreliable (cf. Ensman \& Burrows 1992). Once the photosphere begins to move inward into the expanding envelope, the light curves predicted by SNEC become robust. Models with greater amounts of mass stripped 


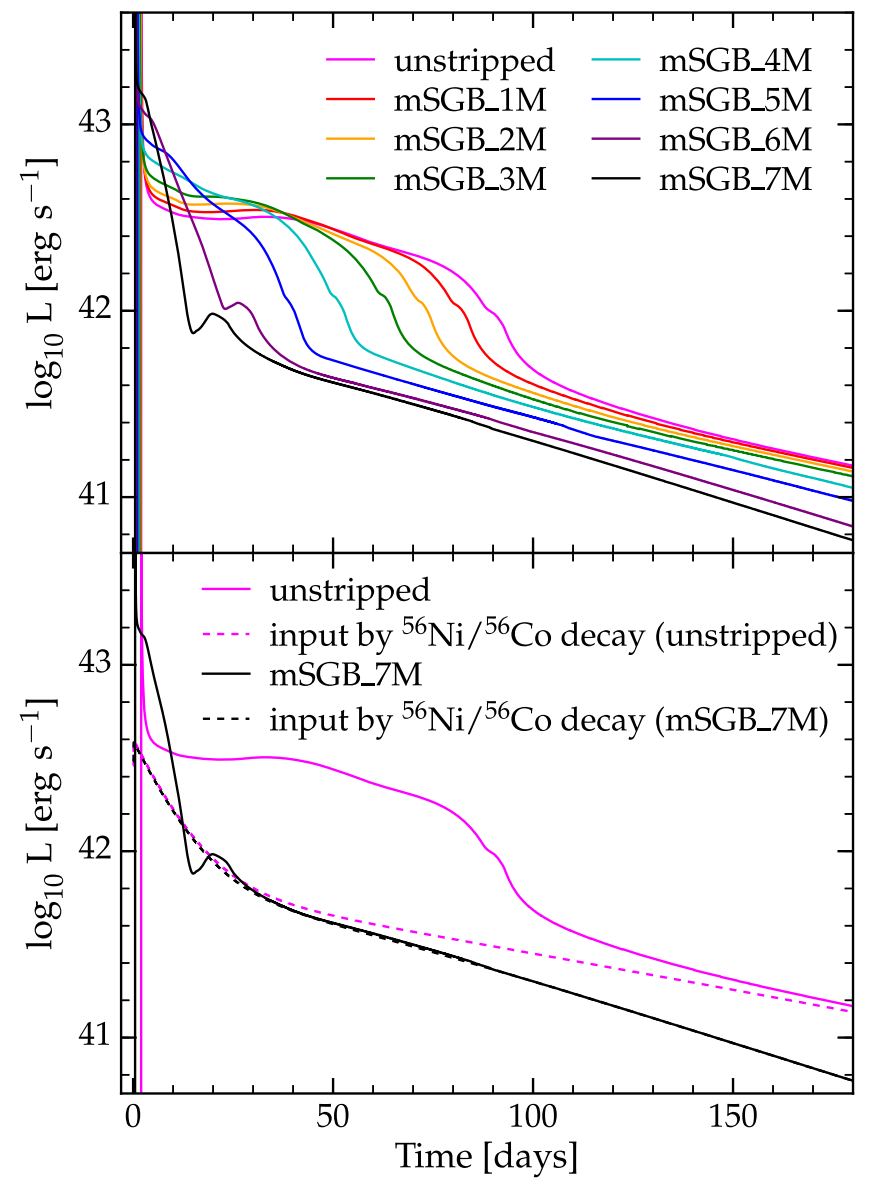

Figure 7. Upper panel: bolometric light curves from the mSGB grid of progenitor models. Lower panel: light curves from the models "unstripped" and "mSGB_7M". Dashed lines show the heating rate from the radioactive decay of ${ }^{56} \mathrm{Ni}$ deposited in each model after taking into account leakage of the gammarays. In the most stripped model, gamma-rays leak out faster than in the unstripped model, impacting the late time light curve.

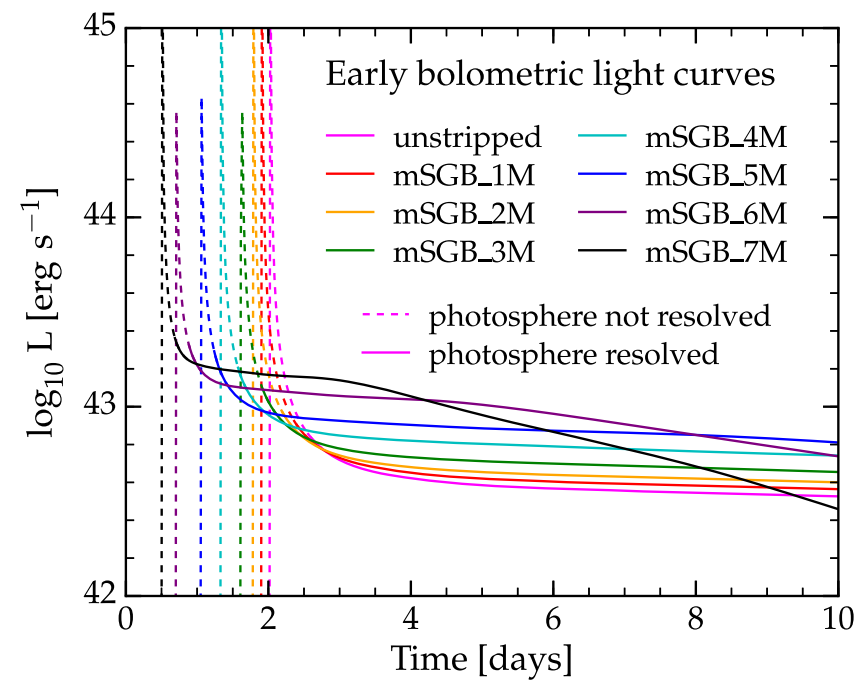

Figure 8. Same bolometric light curves as in Figure 7, but instead focusing on early times around shock breakout. Time is given relative to the onset of the thermal bomb driving the explosion. Solid parts of the curves start from the time at which the photosphere moves inwards in the grid space, while dashed parts indicate that the photosphere is located in the outermost grid cell and is spatially poorly resolved. Note that more stripped models have a higher luminosity in the post-breakout cooling phase and a faster evolving light curve.

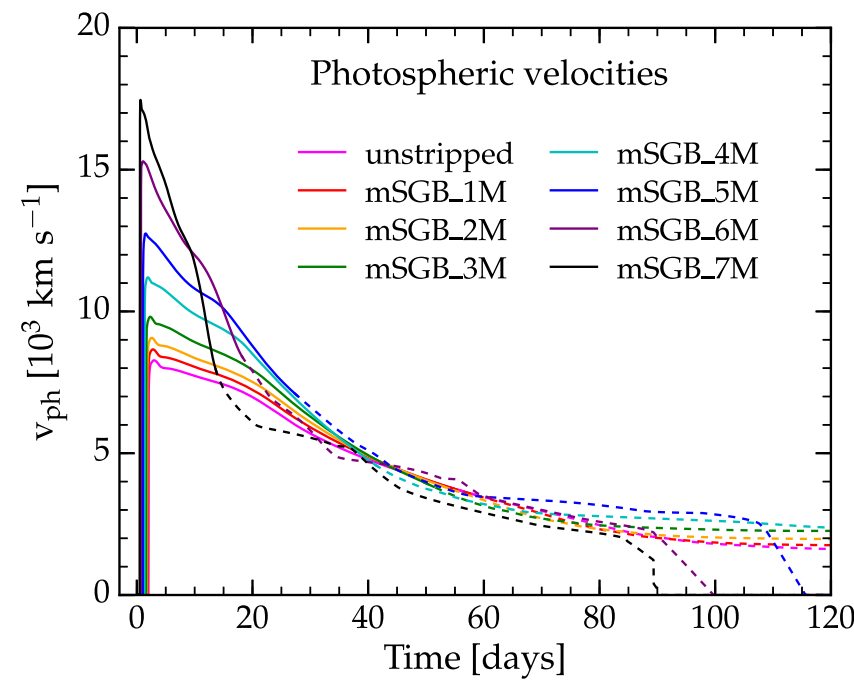

Figure 9. Photospheric velocities $v_{\mathrm{ph}}$ in the mSGB model series, including the unstripped reference model. These velocities are reliable only until the photospheric temperature drops below $T \sim 10^{3.75} \mathrm{~K}$ below which we cannot accurately estimate the location of the photosphere due to low- $T$ opacity limitations in SNEC. We indicate the unreliable part of $v_{\mathrm{ph}}$ by plotting it in dashed lines. Note that the temperature drops most rapidly in the most stripped models that lack a plateau phase (cf. Figure 7).

have higher luminosities in the cooling phase and decay more rapidly. In more stripped models, the $\mathrm{SN}$ shock has to propagate through less envelope mass that is more tenuously distributed. This results in a higher shock velocity at early times as shown in Figure 9 (and earlier breakout; as shown in Figure 8), which leads to a hotter photosphere and a more rapid expansion of the ejecta. This translates directly to a higher initial luminosity and a more rapid decay of the light curve.

From the top panel of Figure 7 we see that as long as there is a substantial amount of hydrogen-rich material left, a clear (if very short) plateau due to recombination can be made out. In our mSGB model series, this is until models mSGB_4M and mSGB_5M, from which we strip $4 M_{\odot}$ and $5 M_{\odot}$ and which have $2.67 M_{\odot}$ and $1.61 M_{\odot}$ of hydrogen-rich material left, respectively. The two most stripped models of this series, models mSGB_6M and mSGB_7M, have only $0.74 M_{\odot}$ and $0.38 M_{\odot}$ of very tenuous hydrogen-rich envelope left, respectively. This does not appear to be sufficient to lead to any plateau and the photosphere recedes very quickly in these models. Instead, models mSGB_6M and mSGB_7M show a clear peak between $\sim 20$ and 30 days that is analogous to the nickel-powered peak that is seen in all types of hydrogendeficient (i.e., Type I) SNe.

Figure 7 shows that stripping of hydrogen-rich envelope mass also has an effect on the late-time radioactively powered part of the light curve. The late-time light curves exhibit changes with mass stripping because there is earlier leakage of gamma-rays from the more highly stripped models. This is shown by the lower panel of Figure 7. It shows the unstripped reference model and the most stripped model $\left(\mathrm{mSGB} \_7 M_{\odot}\right)$ in comparison with the total amount of heating deposited in each model due to the radioactive ${ }^{56} \mathrm{Ni} \rightarrow{ }^{56} \mathrm{Co} \rightarrow{ }^{56} \mathrm{Fe}$ decay chain. The most stripped model has less radioactive heating at late times.

Figure 10 compares the bolometric light curves of models stripped at the mSGB, (the model series we focus on), hMR, and maximum radial extent of the convective envelope (MCE); 


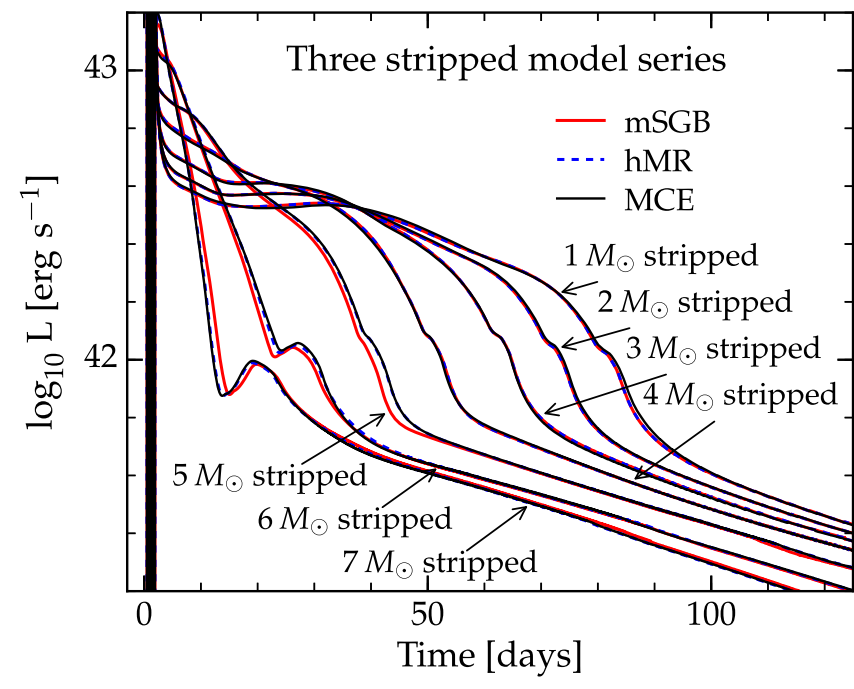

Figure 10. Bolometric light curves for all models from the three different MESA series, described in the Tables 1 and 2. The explosion setup is identical in all cases and as described in Section 4.1. All models are stripped after they have left the main sequence, but the precise point of stripping has little influence on the resulting light curve.

cf. Section 3.1 and Table 1. The light curves of models with the same amount of mass stripped at different times are nearly identical, supporting our initial assertion on the basis of Figure 2. Any variation between light curves of models stripped at mSGB, hMR, and MCE is arguably smaller than the level of systematic uncertainty inherent to SNEC's approximate (i.e., equilibrium diffusion) way of predicting these light curves. The robustness of the light curves suggests that comparisons with observations may allow reliable conclusions about the amount of hydrogen left after (rapid) mass loss events. However, we note that we exclusively consider postMS mass loss. Early large-scale mass loss events on the MS may lead to different outcomes, which should be explored in future work.

In Figure 11, we plot approximate absolute magnitudes of the mSGB series light curves in the IRVB- and $U$-bands and in Figure 12 , we focus on the $V$-band. We obtain the band light curves by assuming blackbody emission from the photosphere and using the bolometric corrections from Ofek (2014). When interpreting these light curves, one should keep in mind two important caveats: (1) When the whole ejecta becomes optically thin, the luminosity has a large contribution of ${ }^{56} \mathrm{Ni} /{ }^{56} \mathrm{Co}$ from above the photosphere. For this reason, we terminate the curves at the points where the luminosity contribution due to ${ }^{56} \mathrm{Ni} /{ }^{56} \mathrm{Co}$ above the photosphere amounts to more than $5 \%$ of the total luminosity. (2) As was demonstrated in Kasen \& Woosley (2009), the $U$ - and $B$-bands of the light curves cannot be adequately reproduced by a onetemperature equilibrium-diffusion code like SNEC, because these bands are strongly influenced by iron group line blanketing after a few tens of days (see, e.g., Figure 8 of Kasen \& Woosley 2009). This causes a much faster decline of the $U$ - and $B$-band light curves. However, the $I R$ - and $V$-bands are still similar to a single temperature blackbody spectrum, and thus these bands are more accurately captured by SNEC.

Finally, we study the sensitivity of our light curves to doubling the explosion energy to $2 \times 10^{51} \mathrm{erg}$ and to doubling the amount of initially present ${ }^{56} \mathrm{Ni}$ to $0.1 M_{\odot}$ in Figure 13 . The qualitative light curve changes are overall as expected from previous work (e.g., Young 2004; Utrobin 2007; Kasen \& Woosley 2009): More energetic explosions have brighter, but faster evolving light curves and an increased amount of ${ }^{56} \mathrm{Ni}$ prolongs the plateau and results in a higher late-time luminosity. Increasing the amount of ${ }^{56} \mathrm{Ni}$ also results in a more pronounced second light curve peak in the most-stripped models mSGB_6M and mSGB_7M. We summarize the connection between these calculations and observed SN light curves in the discussion below.

\section{DISCUSSION AND CONCLUSIONS}

We presented the new open-source SNEC for investigating supernova $(\mathrm{SN})$ explosions and the resulting light curves. SNEC, while currently limited to equilibrium (single temperature) radiative diffusion, is the first such code that is publicly available and this paper will serve as a reference and starting point as SNEC is utilized and improved by both us and the broader community in the future.

As a first application of SNEC, we studied the explosions of $M_{\text {ZAMS }}=15 M_{\odot}$ stars with varying levels of rapid post-MS mass loss at different times in the stars' evolution from the main sequence to the supergiant stage. We evolved these stars to the onset of core collapse with the open-source MESA stellar evolution code and then exploded them with SNEC using a thermal bomb resulting in an asymptotic explosion energy of $10^{51} \mathrm{erg}$. At three different times during the evolution to the supergiant stage, we systematically stripped hydrogen-rich material in units of $1 M_{\odot}$, leaving in the most extreme case only a thin radiative hydrogen-rich layer above the hydrogen shell burning zone. In this experiment in massive star evolution, we find that the time of stripping has essentially no influence on the structure of the envelope and thus most of the SN light curve. Stars with more than $1.5-2 M_{\odot}$ of hydrogen-rich material left die as red supergiants with $R \gtrsim 900 R_{\odot}$ and our most stripped star $\left(0.31 M_{\odot}\right.$ of hydrogen-rich envelope left $)$ dies as a yellow supergiant with a still extended, very tenuous envelope of $R \sim 550 R_{\odot}$.

We find that the time of stripping and the amount of mass stripped has a big but not clearly systematic effect on the structure of the layers immediately surrounding the iron core (mass coordinate $\sim 1.5-3.3 M_{\odot}$ ). The structure in this particular region has been shown to be highly relevant for deciding the ultimate outcome of core collapse (explosion/no explosion, black hole/neutron star remnant; O'Connor \& Ott 2011; Ugliano et al. 2012; Ertl et al. 2015). Our results suggest the need for a detailed study of the sensitivity of presupernova stellar structure to large amounts of rapid mass loss (e.g., via unstable mass transfer in a binary).

The light curves resulting from our set of presupernova models show SN IIP-like morphology for models with more than $\sim 1.5-2 M_{\odot}$ of hydrogen-rich envelope material left at the presupernova stage. The most stripped models $\left(\lesssim 1.5 M_{\odot}\right.$ of hydrogen-rich envelope left) have higher luminosity in the post-breakout cooling phase, but show no plateau, but a second peak around 20-30 days due to energy input from the radioactive decay of ${ }^{56} \mathrm{Ni} /{ }^{56} \mathrm{Co}$ that is uncovered by the rapidly receding photosphere in these models.

In those models that show a plateau in their bolometric light curves, the duration of the plateau phase varies in the range $\sim 20-100$ days (we include both the cooling and the recombination phases of the plateau in the plateau duration; Popov 1993; Kasen \& Woosley 2009), with plateau length decreasing 


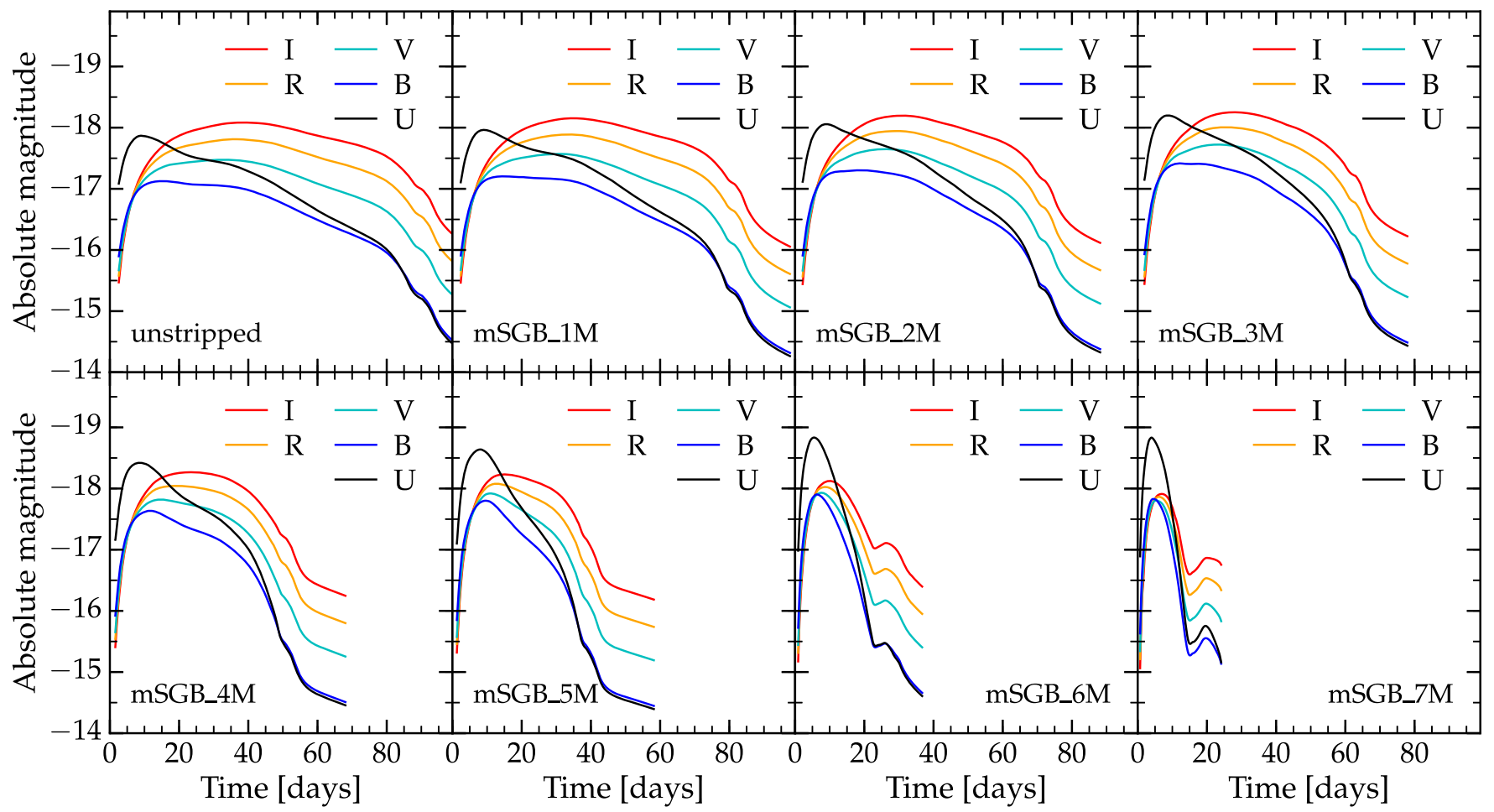

Figure 11. Light curves in absolute magnitude in IRVB and $U$ bands obtained with SNEC for our mSGB model set. The time is given relative to the onset of energy injection by the thermal bomb. The light curves start when the photosphere no longer coincides with the outermost grid cell in the SNEC calculation. Shock breakout, which is in the UV, would be visible in $U$ band, but is not shown. The curves are terminated at the point at which the explosion begins to transition to the nebular phase and the blackbody approximation underlying the band light curves is no longer valid (we define this point at the time at which $5 \%$ of the luminosity comes from above the photosphere due to gamma-ray deposition). We note that real SN light curves fade away in $U$ and $B$ bands considerably faster than predicted by SNEC (e.g., Kasen \& Woosley 2009; Dessart et al. 2013). This is due to line blanketing by iron group elements that is unaccounted for in our models.

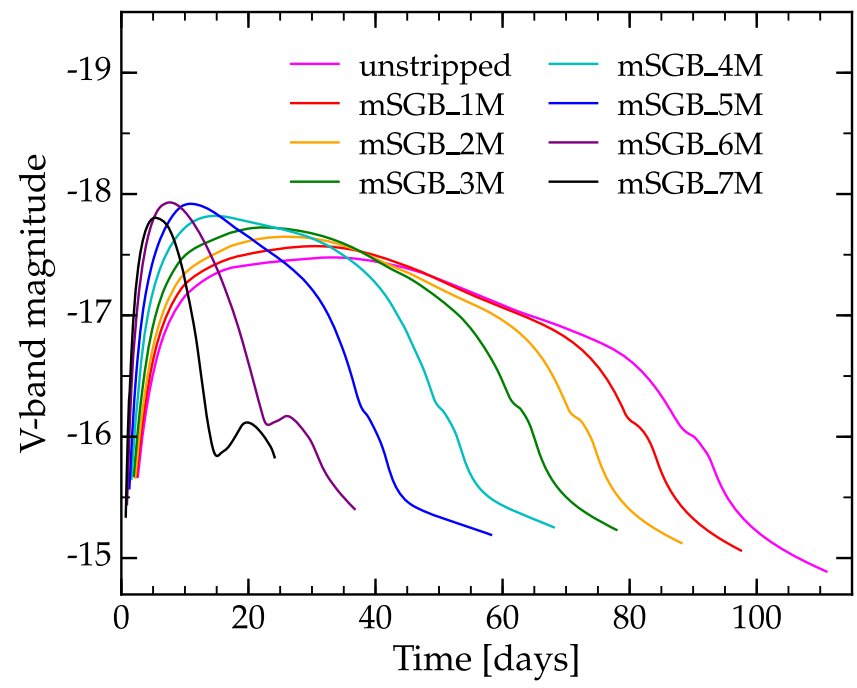

Figure 12. Light curves in absolute $V$-band magnitude obtained with SNEC for the mSGB model set. The time is given relative to the onset of energy injection by the thermal bomb. The $V$-band light curves of more stripped models evolve (rise, decay) faster than those of less stripped models.

with decreasing mass of hydrogen-rich envelope material. In nature, most SNe IIP show plateaus of $\sim 80-100$ days (e.g., Poznanski et al. 2009; Arcavi et al. 2012), although there may be some evidence for a subset of shorter plateaus (Anderson et al. 2014). The completely mass stripped $\mathrm{SNe} \mathrm{Ib} / \mathrm{c}$ show no plateau at all. Both SNe IIP and $\mathrm{SNe} \mathrm{Ib} / \mathrm{c}$ are relevant to our study given the inference that many SN IIP progenitors have ZAMS masses around $\sim 15 M_{\odot}$ (Smartt et al. 2009) and arguments that most $\mathrm{SNe} \mathrm{Ib} / \mathrm{c}$ must come from a similar mass range (Smith et al. 2011). The apparent paucity of observed short and intermediate-length plateaus suggests that in nature hydrogen mass loss is an all or nothing process, at least for the ZAMS mass we consider. This is perhaps not surprising given that for $M_{\text {ZAMS }} \lesssim 20 M_{\odot}$ radiative driven winds are rather weak in normal prescriptions and appreciable mass loss can probably only occur from events like binary interactions, which would not be expected to, say for example, rip off just $\sim 50 \%$ of the mass.

Our most stripped models still have $\approx 0.3-0.4 M_{\odot}$ of hydrogen present and thus should make some connection with $\mathrm{SNe}$ Ilb. Indeed, the light curves of these progenitors show two distinct peaks, similar to the morphology of many SN IIb, where the first peak comes from the shock cooling of the remaining surface hydrogen and the second from radioactive heating (Woosley et al. 1994; Bersten et al. 2012; Nakar \& Piro 2014). However, in detail, the width of the first peak is too large, which is likely due to our models having too much hydrogen still present (Nakar \& Piro 2014). In addition, the second peak in our light curves is sometimes too dim in comparison to observed $\mathrm{SNe}$ IIb due to our models having somewhat less ${ }^{56} \mathrm{Ni}$ (Lyman et al. 2014). However, there is a lot of diversity and uncertainty in the amount of ${ }^{56} \mathrm{Ni}$ produced in $\mathrm{SNe} \mathrm{IIb}$ (see the work and discussions in Shigeyama et al. 1994; Bersten et al. 2012; Ergon et al. 2015), thus we plan to investigate this in more detail in future work.

Another interesting connection to consider is how our results relate to SNe IIL. There has been a long-standing discussion on whether they form a continuous sequence of events that smoothly transition to SNe IIP. The idea that SNe IIL are 


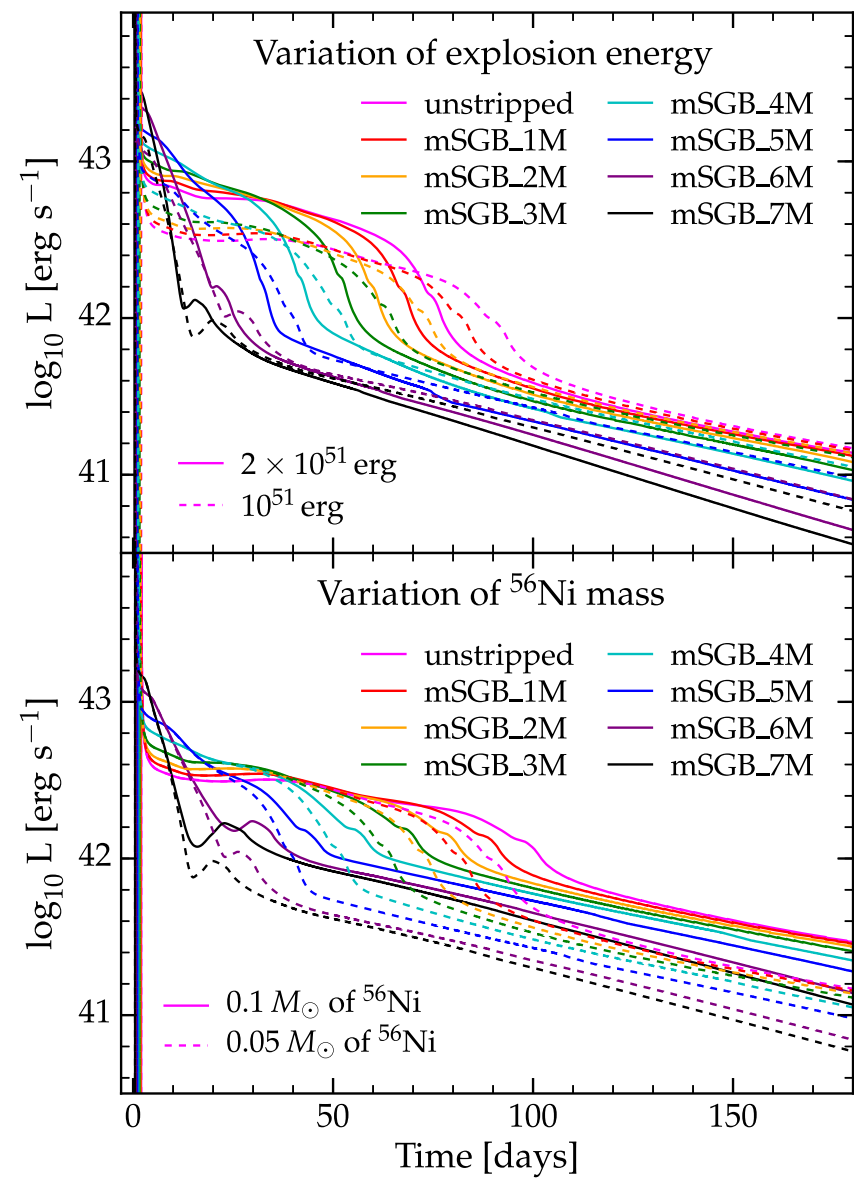

Figure 13. Bolometric light curves for the mSGB model set as in Figure 7, but with variations in explosion energy and ${ }^{56} \mathrm{Ni}$ mass. The time is given relative to the onset of energy injection by the thermal bomb. Top panel: comparison of light curves of models with the fiducial final kinetic energy $\left(10^{51} \mathrm{erg}\right.$, dashed curves) and with twice that energy $\left(2 \times 10^{51} \mathrm{erg}\right.$, solid curves $)$. Increasing the explosion energy leads to brighter, more rapidly evolving explosions in agreement with previous work. Bottom panel: comparison of light curves of models with the fiducial ${ }^{56} \mathrm{Ni}$ mass $\left(0.05 M_{\odot}\right.$, dashed curves $)$ and with twice that amount of ${ }^{56} \mathrm{Ni}\left(0.1 \mathrm{M}_{\odot}\right.$, solid curves $)$. More nickel leads to extended plateaus and brighter radioactive tails. The qualitative changes due to variations in explosion energy and ${ }^{56} \mathrm{Ni}$ mass are in agreement with what was found in previous work (e.g., Young 2004; Kasen \& Woosley 2009).

instead distinct from SNe IIP has been argued for by Arcavi et al. (2012) and Faran et al. (2014), but more recently it has been shown that SNe IIL actually show a significant drop in their light curves at late times ( $\sim 100$ days, Valenti et al. 2015), much like SNe IIP. If there was a continuous range of events from SNe IIL to SNe IIP, then a natural physical mechanism to consider is gradual loss of the outer hydrogen, where SNe IIL would be on the hydrogen-poor side. At least for the ZAMS mass we consider here $\left(15 M_{\odot}\right)$, this does not appear to be the case. First, our results show that intermediate levels of hydrogen mass loss simply shorten the plateau length which is different from SNe IIL, which appear to have roughly normal duration, but steeply declining "plateaus" when they are followed for a sufficient amount of time (Valenti et al. 2015). Second, SNe IIL are on average more luminous than SNe IIP by $\sim 1.5 \mathrm{mag}$ in the optical during the first $\sim 10$ days (Patat et al. 1993, 1994; Anderson et al. 2014; Faran et al. 2014; Sanders et al. 2015). Our more stripped models show slightly higher luminosities at early times, but not nearly extreme enough. Overall, however, our findings, in combinations with recent observations (Valenti et al. 2015), appear to argue that perhaps SNe IIL do not necessarily have less hydrogen, but the hydrogen mass is distributed in a different way. The brighter early light curves would argue that SNe IIL have material at a larger radius (Piro \& Nakar 2013). The occurrence of narrow line features in SNe IIn that might otherwise look somewhat like an SN IIL (Smith et al. 2015) might argue for some contribution from circumstellar material. SNEC is well-suited for addressing these ideas in a systematic way in future work since various mass and density distributions can easily be implemented to investigate what is in fact needed to reproduce SN IIL light curves.

Future work will be directed toward exploiting SNEC's current capabilities for the systematic and reproducible light curve modeling for a broad range of SN explosions, but also toward improving SNEC's transport solver and opacity microphysics. In a first step, we will upgrade SNEC to handle separate radiation and matter temperatures with the long-term goal of constructing an open-source multi-group radiationhydrodynamics code. Of course, input from the community will be especially critical for steering SNEC's further evolution and we look forward to the community's feedback.

We acknowledge helpful discussions with and feedback from J. P. Anderson, W. D. Arnett, M. Bersten, A. Burrows, L. Dessart, C. Fryer, M. Modjaz, E. S. Phinney, D. Radice, S. N. Shore, N. Smith, A. Soderberg, and C. Wheeler. We thank M. Bersten for providing us with the initial conditions necessary to reproduce her results on SN 1999em and also for generously answering our questions. We thank L. Dessart for helping us better understand the discrepancies between SNEC light curves and his results. We thank B. W. Mulligan for helping us to develope the code. Some of the ideas underlying this study were inspired by discussions at Palomar Transient Factory Theory Network workshops at the Sky House, Los Osos, CA. SNEC is available as open source from http:// stellarcollapse.org/SNEC. We thank Frank Timmes for allowing us to use and distribute with SNEC his equation of state and Saha solver routines. We thank the OPAL opacity project, in particular Carlos Iglesisas, allowing us to distribute their interpolation routines and opacity tables with SNEC. We thank Jason Ferguson for allowing us to distribute the lowtemperature opacity tables of Ferguson et al. (2005) with SNEC. This work is supported in part by the National Science Foundation under award Nos. AST-1205732 and AST1212170 , by Caltech, and by the Sherman Fairchild Foundation. The computations were performed on the Caltech compute cluster Zwicky (NSF MRI-R2 award no. PHY0960291), on the NSF XSEDE network under allocation TGPHY100033, and on NSF/NCSA Blue Waters under NSF PRAC award no. ACI-1440083.

\section{APPENDIX A}

\section{COMPARISON WITH BERSTEN ET AL. (2011)}

The work of Bersten et al. (2011) is among the codes that are most similar to SNEC, so it is important to compare light curves. This is done using their progenitor model for SN 1999em, kindly provided by the authors (details of the structure and composition of the model may be found in their work). We use the same explosion energy of $E=1.25 \times 10^{51} \mathrm{erg}$. We also use a step function for the opacity floor, i.e., $0.01 \mathrm{~cm}^{2} \mathrm{~g}^{-1}$ for material with $Z \leqslant 0.3$ and $0.24 \mathrm{~cm}^{2} \mathrm{~g}^{-1}$ for material with 



Figure 14. Left panel: a comparison of bolometric light curves for SN 1999em. The black and red lines show SNEC light curves generated with 1000 and 3000 grid cells, respectively. The blue circles show observational data and the blue curve is the light curve of Bersten et al. (2011), both taken from their Figure 5. The green graph shows the closest light curve we could obtain to the one of Bersten et al. (2011) (see the text for details). The dashed line gives the total power input due to radioactive decay. Right panel: photospheric velocity, calculated with SNEC for the same model and resolutions as in the left panel. The blue circles show observational data and the blue graph shows the result of Bersten et al. (2011), both taken from their Figure 6. Note, that we do not take the opacity floor into account when determining the location of the photosphere, as described in Section 2.2.

$Z>0.3$. This is the only model in this paper where we use this opacity law, since we want to explicitly follow Bersten et al. (2011).

The left panel of the Figure 14 shows bolometric light curves generated with SNEC and the light curve taken from Figure 5 of Bersten et al. (2011), together with the observational data for SN $1999 \mathrm{em}$. The green light curve was generated with SNEC using the original 200-cell grid setup of the model of Bersten et al. (2011). Two other SNEC light curves, which were generated from the same model mapped onto grids with 1000 and 3000 cells, disagree with the 200 grid cells curve at early times as well as during the transition between plateau and ${ }^{56} \mathrm{Ni}$ tail. We have identified two things that may be key contributors to these differences.

First, the differences at early times before the plateau phase may be explained by differences in resolution and the resolution gradient between the 200-cell grid on the one hand, and the 1000-cell and 3000-cell grids on the other hand. To further illustrate the differences in resolution, we plot in Figure 15 the mass resolution as a function of enclosed mass for the three grids. Note that during the first 50 days of the light curve, the photosphere moves inwards from a mass coordinate of $19 M_{\odot}$ (the total mass of the model) to a mass coordinate of $16 M_{\odot}$. The $16-19 M_{\odot}$ region is precisely where Figure 15 shows very large differences in resolution between the different grids. The original 200-cell grid of the double polytropic model of Bersten et al. (2011) has the finest resolution near the surface of the star, but very rapidly changes to the coarsest resolution in the bulk of the model. In fact, we encountered numerical difficulties exploding the 200-cell model. We find that we have to use an outer boundary condition that is different from that described in Section 2.1 of this paper: for numerical stability, we find that we have to impose zero temperature at the surface of the star instead of constant luminosity in the two outermost grid points. The numerical evolutions and light curves from the 1000-cell and 3000-cell runs, however, have negligible dependence on the switch between these two boundary

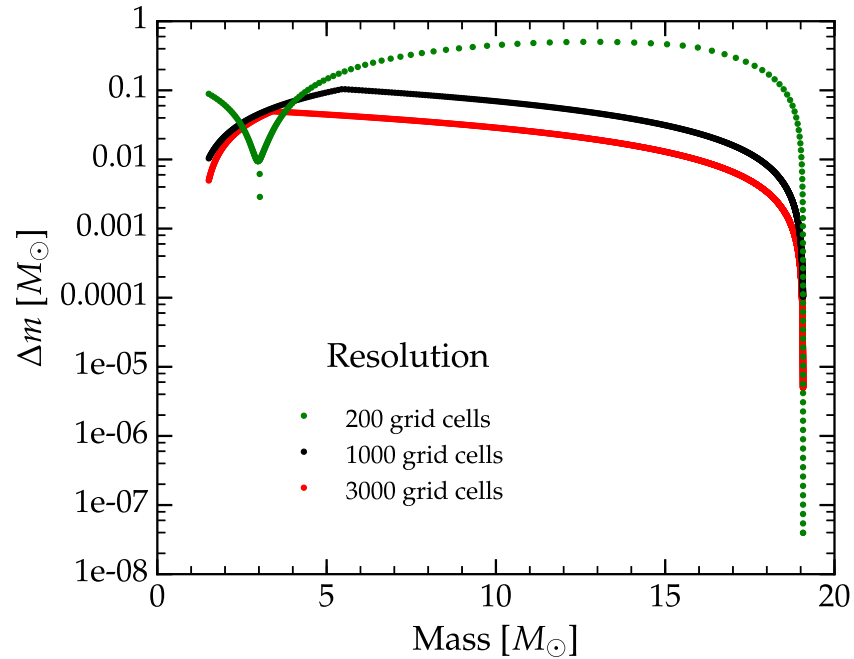

Figure 15. Mass resolution as a function of mass coordinate for the 200, 1000 and 3000 grid cells simulations from Figure 14. The grid having 200 cells is the original grid of the model, which we received from the authors of Bersten et al. (2011).

conditions. Note that the light curves from the 1000-cell and 3000-cell runs lie on top of each other, demonstrating that our SNEC results are numerically converged.

Second, the difference in the transition from the plateau to the ${ }^{56} \mathrm{Ni}$ tail may be explained by the difference in opacities we use for the 200-cell run on one hand, and 1000-cell and 3000cell runs on the other hand. For the 200-cell run, we use opacity tables that are different from those described in Section 2.2: In the low-temperature region $\left(10^{2.7} \mathrm{~K}<T<10^{4.5} \mathrm{~K}\right)$ where OPAL tables are not available, we employ the Ferguson et al. tables (Ferguson et al. 2005) for all densities, temperatures, and compositions. These tables depend on hydrogen mass fraction, density, and temperature, and otherwise assume simply rescaled solar composition. Hence, we ignore the dependence of the opacity on variations of the 
carbon and oxygen mass fractions. We find that this approach is essential for reproducing the light curve of Bersten et al. (2011). Note, however, that these authors do not explicitly state how they treat the opacity in low-temperature, Carbon/Oxygen rich regions. The SNEC light curves generated with 1000 and 3000 grid cells use our standard opacities as described in Section 2.2. As mentioned in that section, the choice of opacity in the low-temperature, high carbon and oxygen mass fractions region weakly influences the evolution of the system, because most of these opacities lie below the opacity floor. However, it does impact the position of the photosphere during the transition between the plateau and the ${ }^{56} \mathrm{Ni}$ tail, as can be seen in Figure 14. We emphasize that the green light curve in the left panel of Figure 14 cannot be reproduced with the standard version of SNEC. We provide it here only to demonstrate how closely we can approach the results of Bersten et al. (2011). Nevertheless, this shows that overall we find reasonable agreement between the light curves shown in Figure 14 and have an understanding of the small differences.

We also compare the velocity evolution calculated with the two codes. The right panel of Figure 14 shows the expansion velocity at the position of the photosphere, calculated with SNEC using the same progenitor model and the grid resolutions as in the left panel of Figure 14. The observational data are taken from Bersten et al. (2011). As in the left panel of Figure 14, the 200 grid cells curve shows the best agreement with Bersten et al. (2011).

As an additional point, it is important to understand whether our treatment applies to light curves at the earliest times. The typical grid setup in SNEC focuses resolution close to the surface of the progenitor star in order to ensure the photospheric region is well resolved as early as possible (as discussed in Section 4.1). A detailed analysis of the very early light curve around shock breakout was carried out by Ensman \& Burrows (1992). In particular, these authors compared the results obtained with a two-temperature radiation-hydrodynamics treatment and with a one-temperature flux-limited equilibrium diffusion treatment similar to SNEC. They concluded that the two approaches give consistent results for light curve and photospheric temperature, provided the surface grid resolution is sufficiently fine so that at the onset of shock breakout a few tens of grid cells are covering the optically thin region outside the photosphere. In our SNEC models, we find that this level of photosphere resolution is not practical for the large model grid we are considering. However, even with our standard gridding, we find that after the first few hours of the explosion that this is sufficient to resolve the photosphere and produce a reliable light curve in the cooling phase after shock breakout.

In order to demonstrate this point, in Figure 16 we plot the early light curves around shock breakout obtained with SNEC using 1000 and 3000 grid cells and the same grid setup as described above for the same progenitor model as in Figure 14. The model with 3000 grid cells always has at least a few grid cells above the photosphere, whereas in the lower-resolution model, the photosphere is remains in the outermost grid cell until it becomes resolved only about one day after breakout. Once this happens, the results of the 1000 and 3000 agree nearly perfectly. But even at earlier times, the two light curves agree surprisingly well. This can be understood from the fact that already shortly after shock breakout the light curve is determined by the diffusion of light from the shock heated

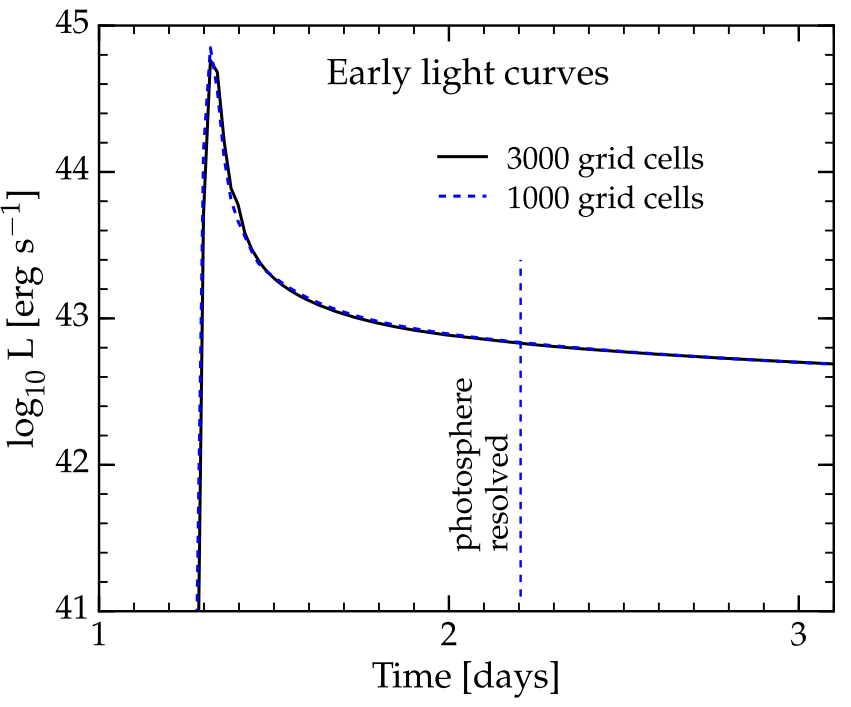

Figure 16. Bolometric light curves around the time of shock breakout using the SN 1999em progenitor model. The black solid and blue dashed curves are SNEC light curves generated with 1000 and 3000 cells, respectively. The higher-resolution model always resolves the photosphere, and although the lower-resolution model does not resolve it until $\sim 2.2$ days, it agrees quite well.

envelope and should not depend anymore on the resolution of the surface layers.

\section{APPENDIX B COMPARISON WITH DESSART ET AL. (2013)}

As an additional comparison, we consider the light curves obtained by Dessart et al. (2013) from a MESA presupernova progenitor. This is exploded with the Lagrangian 1D hydrodynamics code V1D (Livne 1993; Dessart et al. 2010), which is then mapped at day 10 to their non-LTE radiative transfer code CMFGEN (Hillier \& Dessart 2012) that assumes homologous expansion. CMFGEN provides a more detailed treatment of radiative transfer than SNEC. For our specific comparison, we choose model m15Mdot, which was evolved with enhanced mass loss. The set of parameters provided in Dessart et al. (2013) is sufficient to generate a MESA progenitor model with similar but not exactly the same as Dessart et al.'s model m15Mdot. The presupernova hydrogen-rich envelope mass in our version of model m15Mdot is $M_{\mathrm{H}} \sim 8.13 M_{\odot}$ and the model's radius is $R \sim 789 R_{\odot}$. Dessart et al. (2013) find $M_{\mathrm{H}} \sim 7.72 M_{\odot}$ and $R \sim 776 R_{\odot}$. we attribute the differences to our version to the different release versions of MESA used by Dessart et al. (2013) and us. Given the differences in the models (and in the codes), we do not expect perfect agreement.

Our SNEC explosions are triggered by a thermal bomb, while Dessart et al. (2013) use a piston. We tried to match the explosion parameters used by Dessart et al. (2013) (given in their Table 2) as closely as possible. In particular, we excised the inner $1.5 M_{\odot}$ in agreement with the location of the piston in the models of Dessart et al. (2013). We choose the explosion energy in such a way that the final total energy of the model is $1.28 \times 10^{51} \mathrm{erg}$. To achieve this, we inject $1.44 \times 10^{51}$ erginto the innermost $0.02 M_{\odot}$ over a time of $0.1 \mathrm{~s}$. In addition, we include $0.081 M_{\odot}$ of ${ }^{56} \mathrm{Ni}$, initially uniformly distributed in the mass coordinate range $1.5-3.5 M_{\odot}$. We apply boxcar smoothing, as for the other models from our study. Although we do not know the precise distribution of ${ }^{56} \mathrm{Ni}$ in the model of Dessart et al. (2013), the authors indicate that it was not 




Figure 17. Comparison of the bolometric light curve of model m15Mdot of (blue line, Dessart et al. 2013) with the light curve that we obtain with SNEC for a similar progenitor model, the same explosion energy, and the same initial ${ }^{56} \mathrm{Ni}$ mass (black line). The Dessart et al. light curve is generated with their CMFGEN non-LTE radiative transfer code that assumes homologous expansion Hillier \& Dessart (2012).

strongly mixed outwards. We used the same prescription for the opacity floor as for all our other models in this study.

Figure 17 compares our SNEC light curve with the light curve obtained by Dessart et al. (2013) and shown in their Figure 7 . We find encouraging agreement between the two light curves in the first $\sim 80$ days, as well as after day 140 (in the nebular phase). The Dessart et al. plateau is 10-20 days longer than predicted by SNEC. There are a couple of possible reasons for this difference to consider.

On one hand, the two progenitor models are not identical and the Dessart et al. model has less mass (by $\sim 0.4 M_{\odot}$ ) in its hydrogen-rich envelope than our model. However, the higher hydrogen-rich mass should result in a somewhat longer plateau (e.g., Young 2004; Kasen \& Woosley 2009) in our model, which is not what we find in Figure 17. Also, one notes from Figure 17 that the two light curves would agree much better if the Dessart et al. light curve was shifted back in time by $\sim 5$ days. It is not clear what would cause such a shift. However, we point out that we find in our SNEC calculations that at day 10 the expansion is not yet homologous and more internal energy has yet to be converted into kinetic energy of expansion (this point was previously discussed by Dessart \& Hillier 2011). Nevertheless, Dessart et al., who assume homologous expansion, map to their radiative transfer code already at day ten. The consequences of such an early mapping should be explored.

On the other hand (L. Dessart 2015, private communication), the differences may be caused by SNEC's radiation-hydrodynamics treatment, the assumption of LTE, and in the opacities we use (see Section 2). CMFGEN solves the timedependent radiative transfer equations, resolving up to 2000 and more so called super-levels in the frequency domain, which typically represent 5000-10,000 atomic levels (see Hillier \& Dessart 2012). In optically thin regions, CMFGEN uses line opacities and emissivities instead of the Rosseland mean values, used by SNEC. This provides a better description of the effects of iron group line blanketing. In order to further isolate potential culprits we have carried out experiments in which we varied the explosion energy, the degree of ${ }^{56} \mathrm{Ni}$ mixing, and the opacity floor. None of these tests produce light curves that are substantially closer to the Dessart et al. (2013) light curves than what is shown in Figure 17.

\section{REFERENCES}

Anderson, J. P., González-Gaitán, S., Hamuy, M., et al. 2014, ApJ, 786, 67 Arcavi, I., Gal-Yam, A., Cenko, S. B., et al. 2012, ApJL, 756, L30

Arnett, W. D. 1980, ApJ, 237, 541

Arnett, W. D. 1988, ApJ, 331, 377

Aufderheide, M. B., Baron, E., \& Thielemann, F. 1991, ApJ, 370, 630

Baklanov, P. V., Blinnikov, S. I., \& Pavlyuk, N. N. 2005, AstL, 31,429

Barbon, R., Ciatti, F., \& Rosino, L. 1979, A\&A, 72, 287

Bartunov, O. S., Blinnikov, S. I., Pavlyuk, N. N., \& Tsvetkov, D. Y. 1994, A\&A, 281, L53

Bayless, A. J., Even, W., Frey, L. H., et al. 2015, ApJ, 805, 98

Bersten, M. C. 2010, PhD thesis, Univ. Chile

Bersten, M. C., Benvenuto, O., \& Hamuy, M. 2011, ApJ, 729, 61

Bersten, M. C., Benvenuto, O. G., Nomoto, K., et al. 2012, ApJ, 757, 31

Bethe, H. A. 1990, RvMP, 62, 801

Blinnikov, S. I. 1996, AstL, 22, 79

Blinnikov, S. I., \& Bartunov, O. S. 1993, A\&A, 273, 106

Blinnikov, S. I., Eastman, R., Bartunov, O. S., Popolitov, V. A., \& Woosley, S. E. 1998, ApJ, 496, 454

Bruenn, S. W., Mezzacappa, A., Hix, W. R., et al. 2013, ApJL, 767, L6

Cappellaro, E. 2014, in IAU Symp. 296, Supernova Envirnomental Impacts, ed. A. Ray \& R. A. McCray (Cambridge: Cambridge Univ. Press), 37

Chieffi, A., Domínguez, I., Höflich, P., Limongi, M., \& Straniero, O. 2003, MNRAS, 345, 111

Chugai, N. N. 1991, SvAL, 17, 210

de Jager, C., Nieuwenhuijzen, H., \& van der Hucht, K. A. 1988, A\&AS, 72,259

Dessart, L., \& Hillier, D. J. 2011, MNRAS, 410, 1739

Dessart, L., Hillier, D. J., Li, C., \& Woosley, S. 2012, MNRAS, 424, 2139

Dessart, L., Hillier, D. J., Waldman, R., \& Livne, E. 2013, MNRAS, 433, 1745

Dessart, L., Livne, E., \& Waldman, R. 2010, MNRAS, 408, 827

Eastman, R. G., Woosley, S. E., Weaver, T. A., \& Pinto, P. A. 1994, ApJ, 430, 300

Ensman, L., \& Burrows, A. 1992, ApJ, 393, 742

Ergon, M., Jerkstrand, A., Sollerman, J., et al. 2015, A\&A, 580, A142

Ertl, T., Janka, H.-T., Woosley, S. E., Sukhbold, T., \& Ugliano, M. 2015, ApJ, submitted (arXiv:1503.07522)

Falk, S. W., \& Arnett, W. D. 1977, ApJS, 33, 515

Faran, T., Poznanski, D., Filippenko, A. V., et al. 2014, MNRAS, 445, 554

Ferguson, J. W., Alexander, D. R., Allard, F., et al. 2005, ApJ, 623, 585

Filippenko, A. V. 1997, ARA\&A, 35, 309

Foley, R. J., Challis, P. J., Chornock, R., et al. 2013, ApJ, 767, 57

Frieman, J. A., Bassett, B., Becker, A., et al. 2008, AJ, 135, 338

Georgy, C. 2012, A\&A, 538, L8

Goldfriend, T., Nakar, E., \& Sari, R. 2014, ApJ, submitted (arXiv:1404.6313)

Hilditch, R. W. 2001, An Introduction to Close Binary Stars (Cambridge, UK:

Cambridge Univ. Press)

Hillier, D. J., \& Dessart, L. 2012, MNRAS, 424, 252

Iglesias, C. A., \& Rogers, F. J. 1996, ApJ, 464, 943

Inserra, C., Sim, S. A., Wyrzykowski, L., et al. 2015, ApJL, 799, L2

Janka, H.-T. 2012, ARNPS, 62, 407

Karp, A. H., Lasher, G., Chan, K. L., \& Salpeter, E. E. 1977, ApJ, 214, 161

Kasen, D., \& Woosley, S. E. 2009, ApJ, 703, 2205

Kasliwal, M. M., Kulkarni, S. R., Gal-Yam, A., et al. 2010, ApJL, 723, L98

Kasliwal, M. M., Kulkarni, S. R., Gal-Yam, A., et al. 2012, ApJ, 755, 161

Kifonidis, K., Plewa, T., Janka, H.-T., \& Müller, E. 2003, A\&A, 408, 621

Kifonidis, K., Plewa, T., Scheck, L., Janka, H.-T., \& Müller, E. 2006, A\&A, 453,661

Kippenhahn, R., Weigert, A., \& Weiss, A. 2012, Stellar Structure and Evolution (Berlin: Springer)

Leaman, J., Li, W., Chornock, R., \& Filippenko, A. V. 2011, MNRAS, 412, 1419

Levermore, C. D., \& Pomraning, G. C. 1981, ApJ, 248, 321

Litvinova, I. I., \& Nadezhin, D. K. 1983, Ap\&SS, 89, 89

Livne, E. 1993, ApJ, 412, 634

Lyman, J., Bersier, D., James, P., et al. 2014, MNRAS, submitted (arXiv:1406.3667 )

Mezzacappa, A., \& Bruenn, S. W. 1993, ApJ, 405, 669 
Mihalas, D., \& Mihalas, B. W. 1984, Foundations of Radiation Hydrodynamics (New York: Oxford Univ. Press)

Minkowski, R. 1964, ARA\&A, 2, 247

Moriya, T., Tominaga, N., Blinnikov, S. I., Baklanov, P. V., \& Sorokina, E. I. 2011, MNRAS, 415, 199

Nadyozhin, D. K. 1994, ApJS, 92, 527

Nakar, E., \& Piro, A. L. 2014, ApJ, 788, 193

Nakar, E., Poznanski, D., \& Katz, B. 2015, ApJ, submitted (arXiv:1506.07185)

Nakar, E., \& Sari, R. 2010, ApJ, 725, 904

Nomoto, K., Suzuki, T., Shigeyama, T., et al. 1993, Natur, 364, 507

O'Connor, E., \& Ott, C. D. 2011, ApJ, 730, 70

Ofek, E. O. 2014, Astrophysics Source Code Library, ascl:1407.005

Paczyński, B. 1983, ApJ, 267, 315

Patat, F., Barbon, R., Cappellaro, E., \& Turatto, M. 1993, A\&AS, 98, 443

Patat, F., Barbon, R., Cappellaro, E., \& Turatto, M. 1994, A\&A, 282, 731

Paxton, B., Bildsten, L., Dotter, A., et al. 2011, ApJS, 192, 3

Paxton, B., Cantiello, M., Arras, P., et al. 2013, ApJS, 208, 4

Perets, H. B., Gal-Yam, A., Mazzali, P. A., et al. 2010, Natur, 465, 322

Piro, A. L., \& Morozova, V. S. 2014, ApJL, 792, L11

Piro, A. L., \& Nakar, E. 2013, ApJ, 769, 67

Popov, D. V. 1993, ApJ, 414, 712

Poznanski, D., Butler, N., Filippenko, A. V., et al. 2009, ApJ, 694, 1067

Quimby, R. M., Kulkarni, S. R., Kasliwal, M. M., et al. 2011, Natur, 474, 487

Rau, A., Kulkarni, S. R., Law, N. M., et al. 2009, PASP, 121, 1334

Sana, H., de Mink, S. E., de Koter, A., et al. 2012, Sci, 337, 444

Sanders, N. E., Soderberg, A. M., Gezari, S., et al. 2015, ApJ, 799, 208

Shigeyama, T., \& Nomoto, K. 1990, ApJ, 360, 242
Shigeyama, T., Nomoto, K., \& Hashimoto, M. 1988, A\&A, 196, 141 Shigeyama, T., Suzuki, T., Kumagai, S., et al. 1994, ApJ, 420, 341

Smartt, S. J., Eldridge, J. J., Crockett, R. M., \& Maund, J. R. 2009, MNRAS, 395, 1409

Smith, N. 2014, ARA\&A, 52, 487

Smith, N., Li, W., Filippenko, A. V., \& Chornock, R. 2011, MNRAS, 412, 1522

Smith, N., Mauerhan, J. C., Cenko, S. B., et al. 2015, MNRAS, 449, 1876

Sukhbold, T., \& Woosley, S. E. 2014, ApJ, 783, 10

Swartz, D. A., Sutherland, P. G., \& Harkness, R. P. 1995, ApJ, 446, 766

Swartz, D. A., Wheeler, J. C., \& Harkness, R. P. 1991, ApJ, 374, 266

Timmes, F. X., \& Arnett, D. 1999, ApJS, 125, 277

Timmes, F. X., \& Swesty, F. D. 2000, ApJS, 126, 501

Ugliano, M., Janka, H.-T., Marek, A., \& Arcones, A. 2012, ApJ, 757, 69

Utrobin, V. 1993, A\&A, 270, 249

Utrobin, V. P. 2007, A\&A, 461, 233

Valenti, S., Sand, D., Stritzinger, M., et al. 2015, MNRAS, 448, 2608

Vink, J. S., de Koter, A., \& Lamers, H. J. G. L. M. 2000, A\&A, 362, 295

Vink, J. S., de Koter, A., \& Lamers, H. J. G. L. M. 2001, A\&A, 369, 574

Von Neumann, J., \& Richtmyer, R. D. 1950, JAP, 21, 232

Wongwathanarat, A., Müller, E., \& Janka, H.-T. 2015, A\&A, 577, A48

Woosley, S. E. 1988, ApJ, 330, 218

Woosley, S. E., Eastman, R. G., Weaver, T. A., \& Pinto, P. A. 1994, ApJ, 429, 300

Young, P. A., \& Fryer, C. L. 2007, ApJ, 664, 1033

Young, T. R. 2004, ApJ, 617, 1233

Young, T. R., Baron, E., \& Branch, D. 1995, ApJL, 449, L51

Zaghloul, M. R., Bourham, M. A., \& Doster, J. M. 2000, JPhD, 33, 977 